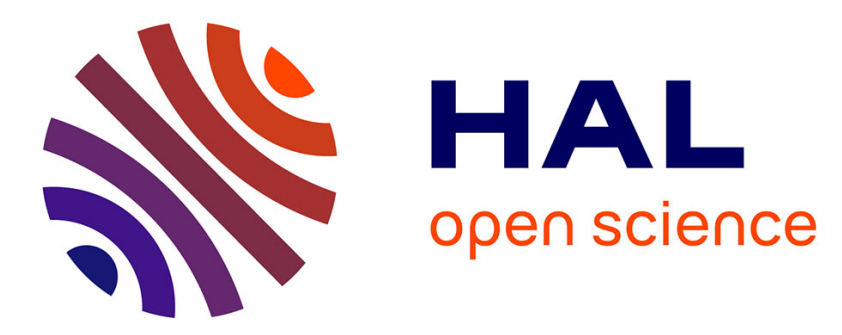

\title{
New lipophenols prevent carbonyl and oxidative stresses involved in macular degeneration
}

Espérance Moine, Manel Boukhallat, David Cia, Nathalie Jacquemot, Laurent Guillou, Thierry Durand, Joseph Vercauteren, Philippe Brabet, Céline

Crauste

\section{To cite this version:}

Espérance Moine, Manel Boukhallat, David Cia, Nathalie Jacquemot, Laurent Guillou, et al.. New lipophenols prevent carbonyl and oxidative stresses involved in macular degeneration. Free Radical Biology and Medicine, 2020, 10.1016/j.freeradbiomed.2020.10.316 . hal-03067208

\section{HAL Id: hal-03067208 https://hal.science/hal-03067208}

Submitted on 15 Dec 2020

HAL is a multi-disciplinary open access archive for the deposit and dissemination of scientific research documents, whether they are published or not. The documents may come from teaching and research institutions in France or abroad, or from public or private research centers.
L'archive ouverte pluridisciplinaire HAL, est destinée au dépôt et à la diffusion de documents scientifiques de niveau recherche, publiés ou non, émanant des établissements d'enseignement et de recherche français ou étrangers, des laboratoires publics ou privés. 
Original article

\title{
New lipophenols prevent carbonyl and oxidative stresses involved in macular degeneration
}

\author{
Espérance Moine ${ }^{\mathrm{a}, *}$, Manel Boukhallat ${ }^{\mathrm{a}}$, David Cia ${ }^{\mathrm{c}}$, Nathalie Jacquemot ${ }^{\mathrm{c}}$, Laurent Guillou ${ }^{\mathrm{b}}$, \\ Thierry Durand $^{\mathrm{a}}$, Joseph Vercauteren ${ }^{\mathrm{a}}$, Philippe Brabet ${ }^{\mathrm{b}}$, Céline Crauste ${ }^{\mathrm{a}, * *}$ \\ ${ }^{a}$ Institut des Biomolécules Max Mousseron (IBMM), Université de Montpellier, CNRS, ENSCM, Montpellier, 34093, France \\ ${ }^{\mathrm{b}}$ Institut des Neurosciences de Montpellier, INSERM U1051, Université de Montpellier, Montpellier, 34091, France \\ ${ }^{\mathrm{c}}$ Laboratoire de Biophysique Neurosensorielle, UMR INSERM 1107, Facultés de Médecine et de Pharmacie, Clermont-Ferrand, 63000, France
}

\section{A R T I C L E I N F O}

\section{Keywords:}

Lipophenol

PUFA

Carbonyl stress

Antioxidant

Macular degeneration

Structure-activity relationship

\begin{abstract}
A B S T R A C T
Dry age-related macular degeneration and Stargardt disease undergo a known toxic mechanism caused by carbonyl and oxidative stresses (COS). This is responsible for accumulation in the retinal pigment epithelium (RPE) of A2E, a main toxic pyridinium bis-retinoid lipofuscin component. Previous studies have shown that carbonyl stress in retinal cells could be reduced by an alkyl-phloroglucinol-DHA conjugate (lipophenol). Here, we performed a rational design of different families of lipophenols to conserve anti-carbonyl stress activities and improve antioxidant properties. Five synthetic pathways leading to alkyl-(poly)phenol derivatives, with phloroglucinol, resveratrol, catechin and quercetin as the main backbone, linked to poly-unsaturated fatty acid, are presented. These lipophenols were evaluated in ARPE-19 cell line for their anti-COS properties and a structureactivity relationship study is proposed. Protection of ARPE-19 cells against A2E toxicity was assessed for the four best candidates. Finally, interesting anti-COS properties of the most promising quercetin lipophenol were confirmed in primary RPE cells.
\end{abstract}

\section{Introduction}

Oxidative stress, resulting from an overproduction of reactive oxygen species (ROS) within cells or in the extracellular matrix, highly damages key cellular proteins, lipids and DNA. Reactive carbonyl species (RCS), such as sugars and osones, endogenous aldehydes or metabolites derived from lipid oxidation, are involved in glycation and cross-linking reactions and thus, affect cellular viability leading to tissue injury. These two carbonyl and oxidative stress (COS) mechanisms play a crucial role in aging-associated pathologies, like age-related macular degeneration (AMD), or in some inherited forms of macular degeneration, such as Stargardt disease [1-3]. AMD is one of the primary causes of central and irreversible visual loss among the elderly in occidental countries, and there is no treatment currently available to stop retinal degeneration, especially in the prevalent dry form ( $80-85 \%$ of cases). COS mechanisms are responsible for the accumulation in retinal pigment epithelium (RPE) of a toxic bis-retinoid conjugate called A2E (its photoisomers and its oxidized metabolites). Pathologic A2E biosynthesis occurs when

Abbreviations: A2E, N-retinylidene-N-retinylethanolamine; ABCA4, ATP binding cassette subfamily A member 4; AGEs, advanced glycation end products; AMD, age-related macular degeneration; ARPE-19, adult retinal pigment epithelial cell line-19; ASAP, atmospheric solids analysis probe; atRAL, all-trans-retinal; BRB, blood retina barrier; BSMR, based on starting material recovery; COS, carbonyl and oxidative stresses; DCC, dicyclohexylcarbodiimide; DCFDA, 2',7'-dichlorofluorescin diacetate; DCM, dichloromethane; DHA, docosahexaenoic acid; DMAP, dimethylaminopyridine; DMEM/F12, Dulbecco's Modified Eagle's Medium (DMEM)/Ham F12; DMF, dimethylformamide; DMSO, dimethylsulfoxide; DNA, deoxyribonucleic acid; EC ${ }_{50}$, efficiency concentration 50; EGCG, epigallocatechin-3-O-gallate; EPA, eicosapentaenoic acid; ESI, electrospray ionization; FBS, fetal bovine serum; 4-HHE, 4-hydroxyhexenal; 4-HNE, 4-hydroxynonenal; HRMS, high resolution mass spectroscopy; LA, linoleic acid; Mp, melting point; MTT, 3-(4,5-dimethylthiazol-2-yl)-2,5-diphenyl tetrazolium bromide; NMR, nuclear magnetic resonance; Nrf2/ Keap1, Nuclear factor (erythroid-derived 2)-like 2/Kelch-like ECH-associated protein 1; PUFA, poly-unsaturated fatty acid; RCS, reactive carbonyl species; $R_{\mathrm{f}}$, retardation factor; ROS, reactive oxygen species; RPE, retinal pigment epithelium; TFA, trifluoroacetic acid; THF, tetrahydrofuran; TIPS, triisopropylsilyl; TLC, thin layer chromatography; UPLC, ultra-performance liquid chromatography; VEGF, vascular endothelial growth factor.

* Corresponding author.

** Corresponding author.

E-mail addresses: esperance.moine@umontpellier.fr (E. Moine), celine.crauste@umontpellier.fr (C. Crauste). 
all-trans-retinal (atRAL), rather than undergoing reduction to retinol in the RPE, accumulates abnormally in photoreceptors. This accumulation can be due to age (AMD) or loss of function of the ABCA4 transporter (Stargardt disease) [2]. Two molecules of this reactive aldehyde (RCS) in excess, condense with one molecule of phosphatidyl ethanolamine (carbonyl stress step) into an unstable dihydropyridinium bis-retinoid. Transformation of the dihydropyridinium bis-retinoid into A2E then arises during the oxidative step leading to a more stable pyridinium form. A2E and its various oxidative metabolites are the major constituent of lipofuscin, a marker of AMD. The massive accumulation of lipofuscin in the RPE (following phagocytosis of photoreceptor outer segments) is cytotoxic and causes progressive RPE cell death and subsequent photoreceptor degeneration [4]. A2E cytotoxicity is explained by several mechanisms: loss of membrane integrity due to its amphiphilic properties during membrane integration; increase of oxidative stress by the generation of singlet oxygen during exposition to blue light; metabolization in A2E-oxidized metabolites resulting in DNA lesions [5-7]. Limitation of A2E biosynthesis (by clearance of atRAL) and oxidation is therefore an attractive target to slow the progression of macular degeneration [8].

Based on epidemiology studies, natural plant antioxidants, such as (poly)phenols, secondary metabolites that protect plants from several aggressions, are efficient at protecting animal organisms against oxidative stress. Some (poly)phenol structures have been reported to protect a variety of retinal cell types from oxidative stress-induced cell death [9-11]. Such activity may be related to their capacity to scavenge directly excess ROS, or to stimulate the enzymatic antioxidant defenses of the organism through Nrf2/Keap1 signaling pathway [12,13]. Moreover, recent literature addressed the efficiency of (poly)phenols to inhibit Advanced Glycation End product formation (AGEs), which result from both carbonyl and oxidative stresses $[14,15]$, and to act also as anti-carbonyl stress derivatives by trapping toxic RCS (glyoxal, acrolein, 4-hydroxynonenal (4-HNE)) [16-18].

Unfortunately, while showing interesting in vitro protection, the limited bioavailability of most (poly)phenols (weak drug absorption, high systemic metabolization, restricted cell penetration ...) negatively influences their in vivo potency and thus their development as drug candidates. Increasing the lipophilicity of those (poly)phenols may be a useful way to improve i) their protective effect on lipid membranes or lipid derivatives (such as A2E), ii) their absorption [19], and iii) their formulation for in vivo administration [20-22]. In our previous work, we synthesized lipophenols (or phenolipids), polyunsaturated fatty acid (PUFA) linked to alkyl-phloroglucinol (LEAD A/B, Fig. 1), that were designed to reduce carbonyl stress associated with retinal dystrophies $[23,24]$. The main objective of those lipophenols was to avoid/slow the pace of A2E formation by scavenging atRAL (Fig. 1). To increase lipophilicity and cellular permeability of starting (poly)phenol, PUFAs were preferred to saturated ones, due to their observed benefits in an
AMD clinical trial [25]. Both isopropyl-phloroglucinols linked to linoleic acid (C18:2 n-6; LA - LEAD A, Fig. 1) and docosahexaenoic acid (C22:6 n-3; DHA - LEAD B, Fig. 1) showed promising protection of RPE cells against atRAL toxicity. A isopropyl functional group was rationally selected to increase the nucleophilic properties of the aromatic cycle involved in the trapping of aldehyde function of atRAL and leading to the formation of a stable non-toxic chromene adduct (Fig. 1) [23,26]. Both isopropyl and PUFA parts were proven to be indispensable in a phloroglucinol series (LEAD A and LEAD B) to ensure cell protection against carbonyl stress in cellular assays [23,24]. The DHA analogue (LEAD B) was selected for further in vivo evaluations due to several benefits of this omega-3 lipid: i) the high proportion of DHA in the membrane of photoreceptor outer segments, ii) its ability to reach the retina through PUFA transports [27]; iii) recent data showing that dietary supplementation with high doses of DHA/EPA significantly improves the visual acuity of AMD patients [25]; and iv) the report that specific oxidative metabolites of DHA (Neuroprotectin D1) protect RPE cells against oxidative stress [28]. Intravenous administration of LEAD B allowed photoreceptor protection against acute light-induced degeneration in a mouse model used for the development of novel therapeutics for Stargardt disease (Abca4 $4^{-/-}$mice) $[29,30]$. Despite interesting preliminary in vivo protection observed using LEAD B, this phloroglucinol derivative lacks efficiency to reduce ROS production and cellular oxidative stress. In the present study, we report new potent alkyl-lipophenols that act on both carbonyl and oxidative stresses, to reduce atRAL toxicity, and oxidation associated with A2E formation, metabolism and toxicity, critical steps involved in photoreceptor degeneration.

Several possibilities have been proposed to increase antioxidant activities while preserving anti-carbonyl stress properties. First, using the same (poly)phenol backbone, PUFA was linked directly to the aromatic cycle to free one phenolic function of the phloroglucinol involved in the antioxidant activity (C-phloroglucinol series, Fig. 1). Second, new series of lipophenols were also developed from natural (poly)phenols, different from phloroglucinol, meeting the criterion that the starting (poly)phenol contained an isopropyl-resorcinol framework mimicking the phloroglucinol backbone, as this was indispensable for the protection against carbonyl stress [23,24]. More effective natural antioxidants, such as resveratrol stilbenoid (true vinylogous analogue of phloroglucinol), or flavonoids, such as quercetin or catechin, all of which were reported to trap aldehyde function in cell free assays [18,31,32] and to protect ARPE-19 cells against A2E photo-oxidation damage [33-35], have been proposed as new anti-COS alkyl-lipophenol series (Fig. 1). Nine novel alkyl-lipophenol-PUFAs have been synthesized using original chemical strategies. Toxicity assays, protection against a lethal concentration of atRAL and reduction of $\mathrm{H}_{2} \mathrm{O}_{2}$ induced ROS production, were used to first screen these molecules in ARPE-19 cells. The necessity of the isopropyl and the PUFA parts for carbonyl stress protection has

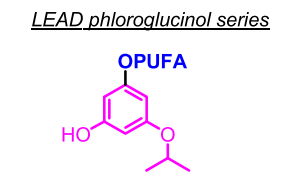

LEAD A: PUFA = linoleic acid (LA)

LEAD A: PUFA = linoleic acid $(\mathrm{LA})$
LEAD B: PUFA = docosahexahenoic acid $(\mathrm{DHA})$
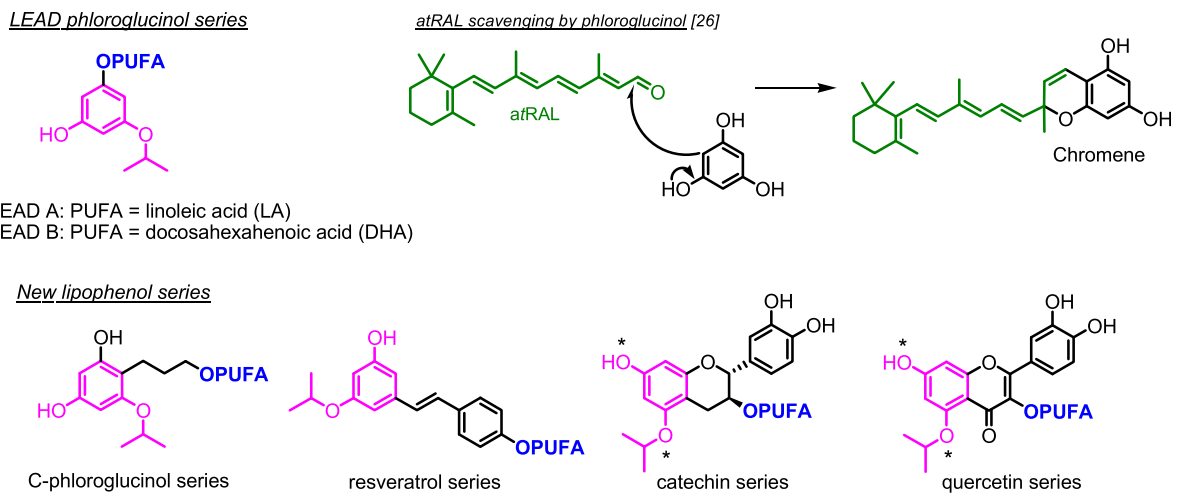

* : interchangeable positions for isopropyl function

Fig. 1. Chemical structures of developed lipophenol series. 
been highlighted with all new (poly)phenol backbones (resveratrol, catechin and quercetin). The ability to preserve antioxidant properties, despite reduction of free phenolic functions compared to native (poly) phenols, has been studied. Best DHA-lipophenol conjugates offering both oxidative and carbonyl stress protection, were compared with LEAD B, for their ability to reduce A2E toxicity during photo-oxidation process in RPE. According to the comparison of both carbonyl and oxidative stress protection, a new alkyl-quercetin-PUFA conjugate has been highlighted. Cell protection was finally validated with this lipophenol in primary RPE cells, as primary cultures are likely to reflect in vivo cell morphology and function more accurately.

\section{Results and discussion}

\subsection{Synthesis and anti-COS evaluation of C-phloroglucinol derivatives}

The first chemical changes considered to increase antioxidant capacity concerned the link of the PUFA moiety. Indeed, releasing one more phenolic function on the phloroglucinol (P) backbone should allow better ROS scavenging properties. This modification was investigated through a $C$-alkylation on the phloroglucinol aromatic ring, by the introduction of a hydroxylated-alkyl spacer, in order to perform PUFA functionalization. The synthesis of the two C-linker analogues $(\mathbf{8 a} / \mathbf{b})$ of LEAD A (P-OiP-OLA) and LEAD B (P-OiP-ODHA) is described in Scheme 1.

Starting from mono-isopropyl phloroglucinol $\mathbf{1}$ [23], a Gattermann-Koch formylation [36] lead to salicylic aldehydes $\mathbf{2 a / 2 b}$ $(80 / 20)$ in $64 \%$ combined yield. The next Wittig reaction was performed on the mixture of $\mathbf{2} \mathbf{a} / \mathbf{2} \mathbf{b}$ for a two-carbon homologation, and the resulting two $\alpha, \beta$-unsaturated esters $\mathbf{3 a} / \mathbf{3} \mathbf{b}$ were reduced under hydrogen in the presence of $\mathrm{Pd} / \mathrm{C}$ catalyst which after purification lead to the isolation of isomer $4 a$. Phenolic functions were then protected by silylated protecting groups (compound 5). The alcohol 6 was obtained after the ester reduction and was coupled either with LA or DHA using classical Steglich coupling conditions. Finally, LA and DHA desired conjugates (respectively $\mathbf{8 a}$ and $\mathbf{8 b}$ ) were obtained after deprotection of silylated phenolic function in the presence of $\mathrm{NEt}_{3} / 3 \mathrm{HF}$.

In vitro evaluations of the $\mathrm{C}$-phloroglucinol derivatives were performed on the ARPE-19 cell line. All compounds were tested at various concentrations in the range of $0-160 \mu \mathrm{M}$ for cytotoxicity assessment, and in the range of $0-80 \mu \mathrm{M}$ for anti-COS evaluation. Regarding the new C-phloroglucinol derivatives ( $\mathbf{8 a}$ and $\mathbf{8 b}$ ), our main concerns were to maintain anti-carbonyl stress activity, despite the reduction of one nucleophilic $C$-alkylation site (on the aromatic cycle) compared to LEAD $\mathrm{A} / \mathrm{B}$ structures, and to increase antioxidant properties by releasing a phenolic function. As shown in Fig. 2, there is no apparent cytotoxicity due to the modification of the lipid position.

Anti-carbonyl stress activity of C-phloroglucinol derivatives is evaluated by comparing cell survival in presence of a toxic concentration of carbonyl stressor atRAL, and is represented in Fig. 3A. The two new Cphloroglucinol derivatives (8a and $\mathbf{8 b}$ ) displayed interesting protective properties, increasing cell viability (at $80 \mu \mathrm{M})$ of $+25 \%$ for LA derivative (8a) and $+28 \%$ for DHA derivative ( $8 \mathbf{b}$ ), compared to non-treated and atRAL-exposed cells. No statistical differences in cell protection were observed under these conditions between P-OiP-OLA (LEAD A) versus POiP-CLA (8a) and between P-OiP-ODHA (LEAD B) versus P-OiP-CDHA (8b), at equivalent concentrations. Modification of the PUFA position on the phloroglucinol backbone did not affect the anti-carbonyl stress activity of the derivative: the PUFA moiety can be introduced indifferently on a phenolic function or with a spacer on the aromatic core. This result is in agreement with the literature, as phloretine, a natural

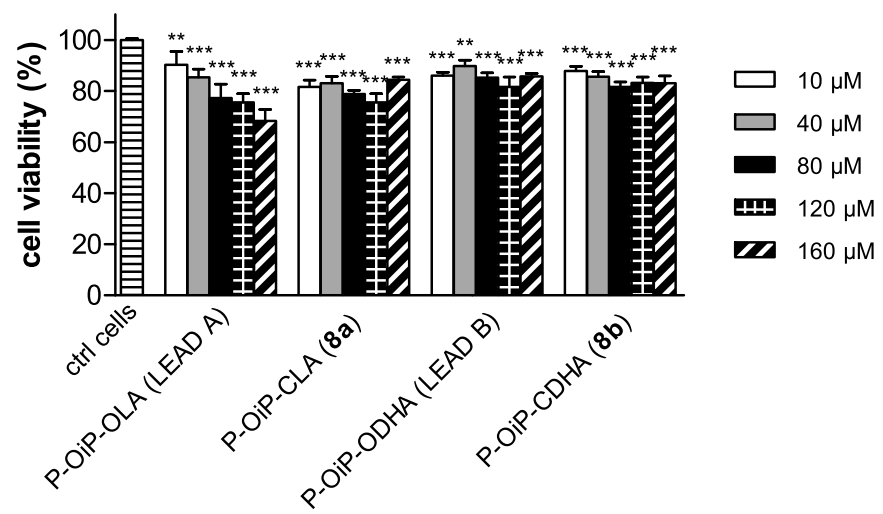

Fig. 2. Evaluation of cytotoxicity of C-phloroglucinol derivatives; P-OiP-OLA (LEAD A) [24], P-OiP-CLA (8a), P-OiP-ODHA (LEAD B) [23] and P-OiP-CDHA (8b). Results are expressed in mean \pm SEM and are from $n=3-5$ independent experiments. ARPE-19 cell viability (MTT) after incubation of phloroglucinol derivatives $(0-160 \mu \mathrm{M})$. The data are expressed as the percentage of non-treated control cells. * $\mathrm{p}<0.05,{ }^{* *} \mathrm{p}<0.01,{ }^{* * *} \mathrm{p}<0.001$, versus non-treated control cells.

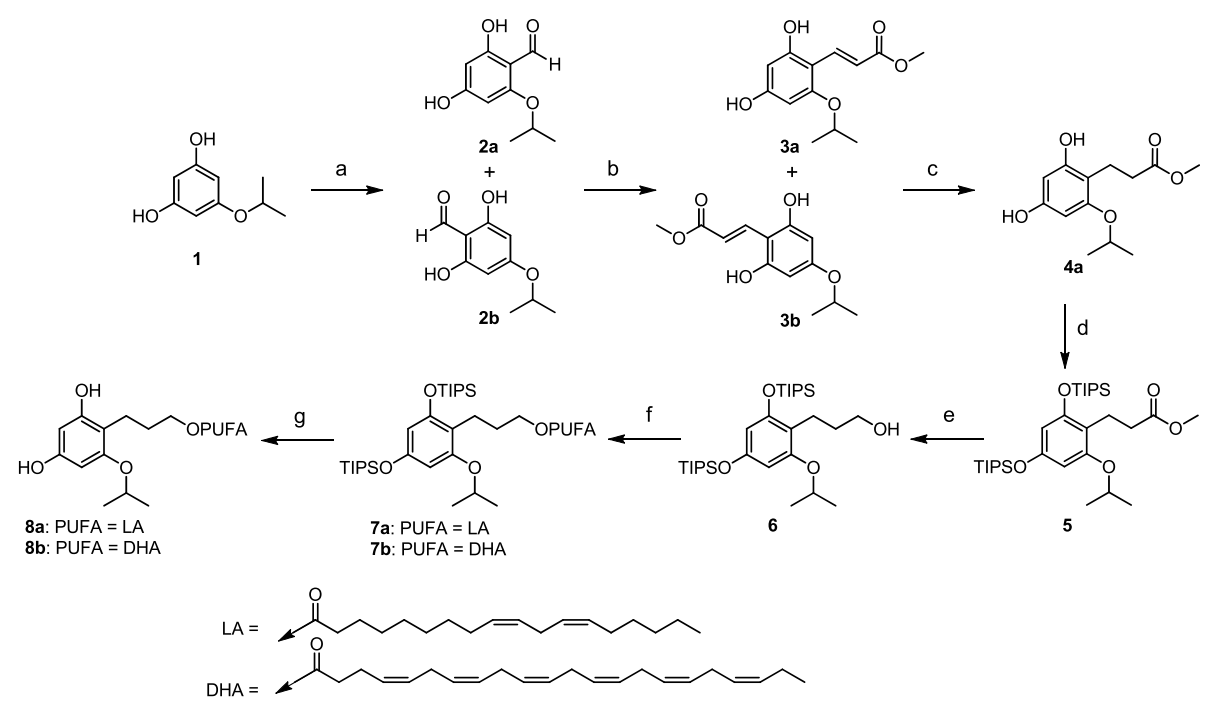

Scheme 1. Chemical pathway leading to C-phloroglucinol derivatives. Reagents and conditions: (a) $\mathrm{Zn}\left(\mathrm{CN}_{2}, \mathrm{ZnCl}_{2}, \mathrm{Et}_{2} \mathrm{O}-\mathrm{HCl}(2 \mathrm{~N}), \mathrm{rt}, 16 \mathrm{~h}\right.$ and $\mathrm{H}_{2} \mathrm{O}, \mathrm{reflux}, 20$ min or $\mathrm{POCl}_{3}$, EtOAc, DMF, rt, 86 h, 64\%; (b) $\mathrm{Ph}_{3} \mathrm{PCHCOOMe,} \mathrm{DCM,} \mathrm{rt,} 18$ h, 59\%; (c) $\mathrm{H}_{2}$, Pd/C, EtOAc, rt, 4 h, 71\%; (d) TIPS-OTf, NEt 3 , DCM, rt, 2 h, 35\%; (e) $\mathrm{LiAlH}_{4}(1 \mathrm{M}), \mathrm{Et}_{2} \mathrm{O}, 0^{\circ} \mathrm{C}, 4 \mathrm{~h} 30,77 \%$; (f) PUFA, DCC, DMAP, DCM, rt to $50{ }^{\circ} \mathrm{C}, 7 \mathrm{~h}, 7 \mathrm{a}: 66 \%$ and $7 \mathbf{b}: 28 \%$; $(\mathrm{g}) \mathrm{NEt}{ }_{3} / 3 \mathrm{HF}, \mathrm{THF}, \mathrm{rt}, 19 \mathrm{~h}, 8 \mathrm{aa}: 39 \%$ and $8 \mathbf{b}: 30 \%$. 
(A)

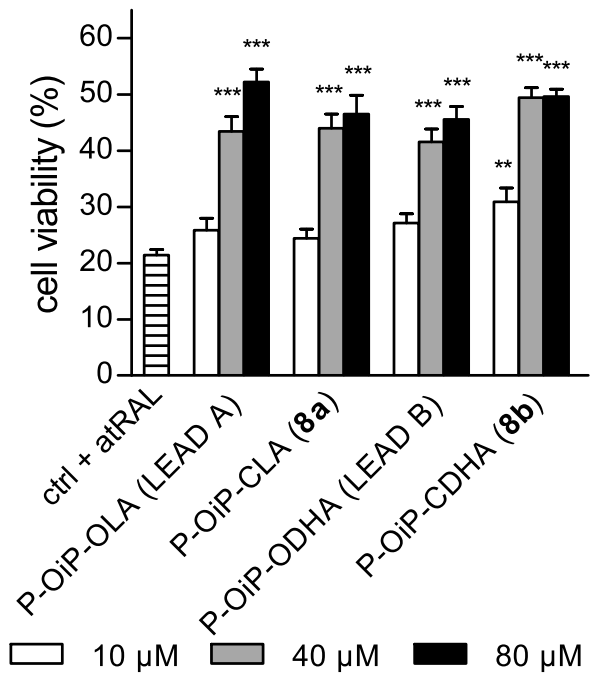

(B)

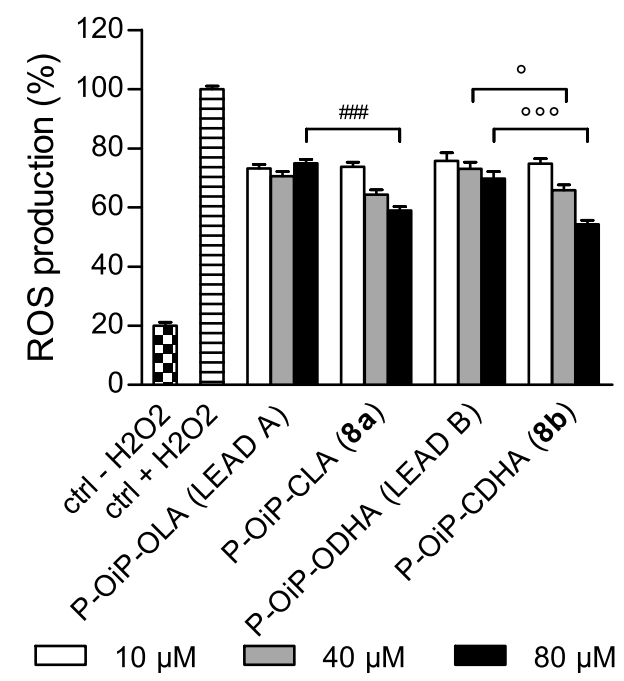

Fig. 3. In vitro anti-COS evaluation of Cphloroglucinol derivatives. Comparison of $\mathrm{P}$ OiP-OLA (LEAD A) [24], P-OiP-CLA (8a), P-OiP-ODHA (LEAD B) [23] and P-OiP-CDHA (8b) activities. Results are expressed in mean \pm SEM and are from $n=$ 3-5 independent experiments. (A) Anti-carbonyl stress assay: ARPE-19 cell viability (MTT) after incubation of phloroglucinol derivatives $(0-80 \mu \mathrm{M})$ and atRAL $(15 \mu \mathrm{M})$. The data are expressed as the percentage of non-treated and non-exposed to atRAL control cells. * $\mathrm{p}<0.05,{ }^{* *} \mathrm{p}<0.01$, $* * * \mathrm{p}<0.001$, versus non-treated and exposed to atRAL cells. (B) Antioxidant assay: representation of ROS production (DCFDA probe) after incubation of phloroglucinol derivatives $(0-80 \mu \mathrm{M})$ and $\mathrm{H}_{2} \mathrm{O}_{2}$ $(600 \mu \mathrm{M})$ in ARPE-19 cells. The data are expressed as the percentage of non-treated and exposed to $\mathrm{H}_{2} \mathrm{O}_{2}$ cells. All conditions have a p-value $<0.001$ versus non-treated and exposed to $\mathrm{H}_{2} \mathrm{O}_{2}$ control cells. $\# \mathrm{p}<$ 0.05 , \#\# $<<0.01$, \#\#\#p $<0.001$, versus P-OiP-OLA (LEAD A) at the same concentration. ${ }^{\circ} \mathrm{p}<0.05,{ }^{\circ} \mathrm{p}<0.01,{ }^{\circ 0} \mathrm{p}<0.001$, versus P-OiP-ODHA (LEAD B) at the same concentration.

phloroglucinol analogue with a substituent on the aromatic ring, is also able to trap toxic aldehydes, such as acrolein, a toxic aldehyde found in cigarette smoke and involved in AMD via induction of oxidative damage in RPE cells, or 4-HNE, derived from lipid peroxidation [31].

The main objective of the synthesis of compounds $\mathbf{8} \mathbf{a}$ and $\mathbf{8 b}$ was to increase antioxidant properties of the LEADs A and B in cell experiments. As expected, $\mathbf{8 a}$ and $\mathbf{8 b}$ logically showed weaker antioxidant activity compared to phloroglucinol (data not shown). Compared to untreated cells, C-phloroglucinol analogues were able to decrease $\mathrm{H}_{2} \mathrm{O}_{2}$ induced ROS production by $41 \%$ for LA derivative (8a) and $46 \%$ for DHA derivative (8b) at $80 \mu \mathrm{M}$ (Fig. $3 \mathrm{~B}$ ). Thus, a significant improvement in antioxidant activity was observed using C-phloroglucinols $(\mathbf{8 a} / \mathbf{8 b})$ that show a dose-dependent effect compared to LEAD A and B. Direct hydroxyl radical scavenging activity of phloroglucinol has been previously reported [26,37], and the liberation of one phenolic function explains the increased antioxidant efficacy of C-phloroglucinol derivatives (8a and $\mathbf{8 b}$ ) compared to LEAD A and LEAD B, that reduce ROS production by only $25 \%$ and $30 \%$ at $80 \mu \mathrm{M}$, respectively.

\subsection{Synthesis and anti-COS evaluation of resveratrol lipophenol analogues}

Resveratrol (R), a major active phytoalexin from the stilbene family (3,4',5-trihydroxystilbene), is mainly found in Vitis vinifera (Vitaceae) stalks and in the roots of Fallopia japonica var. japonica (Polygonaceae). Resveratrol has a wide range of biological activities including antibacterial and anti-fungal properties [38], antioxidant activity [10,39], and anti-inflammatory properties [40]. Furthermore, previous evaluations on RPE cell assays highlighted the benefit of resveratrol in reducing VEGF effect and increasing natural antioxidant enzymatic and molecular defenses [40]. Resveratrol should not only be considered as a simple ROS scavenger. The work of Vlachogianni et al. shows better radical scavenging activity of $4^{\prime}$-acetylated resveratrol compared to 3-acetylated analogues [41]; we decided therefore to link the PUFA at the $4^{\prime}$ position of resveratrol. The isopropyl moiety was introduced at the 5 position in order to keep the alkyl-resorcinol moiety, which was proven to be responsible for high anti-carbonyl stress activity on phloroglucinol series [23]. In order to confirm the necessity of alkyl as well as PUFA moieties on the resveratrol backbone, as we did previously with phloroglucinol [23,24], we produced in addition to alkyl resveratrol-PUFA $(\mathbf{1 5 a} / \mathbf{b})$, an isopropyl-resveratrol derivative not linked to PUFA (R-5OiP 13, Scheme 2) and derivatives linked to PUFAs without alkyl function named resveratrol-4'LA (R-4'LA) and resveratrol-4'DHA (R-4'DHA), both synthesized as previously described [42, 43].

A tedious step to access the desired alkylated resveratrols $15 a / b$, was the isopropylation of the 5 position of resveratrol as the $4^{\prime}$ position is the more reactive. Utilization of the supported lipase Novozyme 435 (Immobilized lipase from Candida antarctica) was efficient and selective to protect the $4^{\prime}$ position of resveratrol, using vinyl butyrate $(52 \%)$. Alkylated compound 10 was then obtained using diisopropylsulfate and $\mathrm{K}_{2} \mathrm{CO}_{3}$ at room temperature for $24 \mathrm{~h}$ [44]. Successive protection of the resulting phenol with TIPS groups, and deprotection of the butyrate in position 4' using ammonia solution in $\mathrm{MeOH}$, gave access to compound
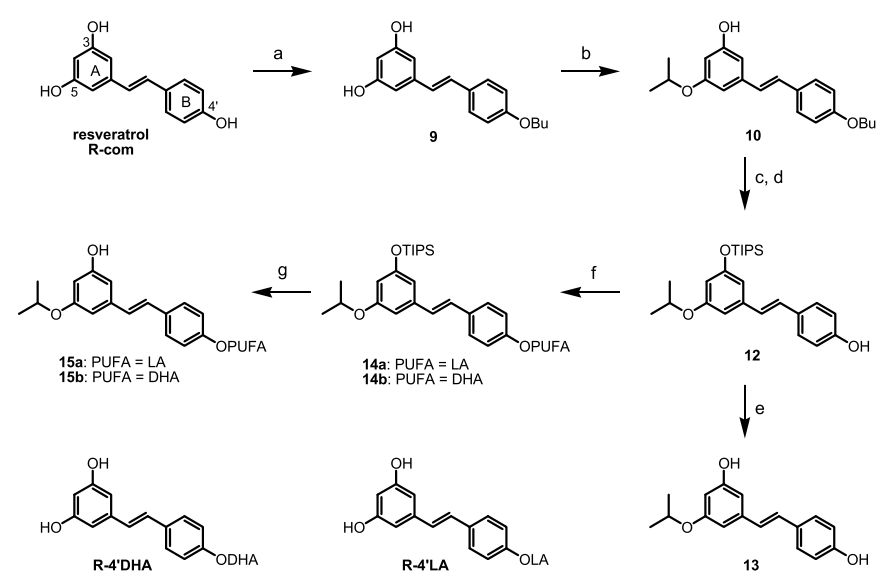

Scheme 2. Synthesis and chemical structures of resveratrol derivatives. Reagents and conditions: (a) 2-methyl-butan-2-ol, vinyl butyrate, Novozyme 435 (CALB), $40{ }^{\circ} \mathrm{C}, 8$ days, $52 \%$; (b) diisopropylsulfate, $\mathrm{K}_{2} \mathrm{CO}_{3}$, acetone, rt, $24 \mathrm{~h}$, $22 \%$ (67\% BSMR); (c) TIPS-OTf, DIPEA, THF, rt, 6 h, 63\%; (d) $\mathrm{NH}_{3} / \mathrm{MeOH}$, DCM, 0 to $5{ }^{\circ} \mathrm{C}, 24 \mathrm{~h}, 80 \%$; (e) $\mathrm{NEt}_{3} / 3 \mathrm{HF}$, THF, rt, $23 \mathrm{~h}, 67 \%$; (f) PUFA, DCC, DMAP, DCM, rt, 14a: $35 \mathrm{~min}, 83 \%$ and 14b: 2 h, 46\%; (g) $\mathrm{NEt}_{3} / 3 \mathrm{HF}$, THF, rt, 15a: 19 h, 67\% and 15b: 20 h, 64\%. 
12, which could be esterified by LA or DHA through classical Steglish conditions, or desilylated to give the desired isopropylated resveratrol (R-5OiP, 13). Final desilylation of the esterified compounds (14a and 14b) gave access to the desired LA- and DHA-isopropylated resveratrol derivatives $\mathbf{1 5 a}$ and $\mathbf{1 5 b}$ using mild $\mathrm{NEt}_{3} / 3 \mathrm{HF}$ reagent.

First, cytotoxicity of resveratrol derivatives on ARPE-19 cell line was evaluated and is represented in Fig. 4. No dose dependent toxicity was observed for LA or DHA alkyl-resveratrol $15 \mathrm{a} / \mathrm{b}$ up to $160 \mu \mathrm{M}$. Introduction of only the isopropyl moiety on resveratrol (R-5OiP, compound 13), led to highly toxic effects from $80 \mu \mathrm{M}$. Interestingly, association of LA or DHA and isopropyl moieties (compounds 15a and 15b) resulted in absent or weak toxicity up to $160 \mu \mathrm{M}$, suggesting a stability of the lipophenol ester during the toxicity assay. Indeed, if the ester link was easily cleaved, the release of the remaining R-5OiP (13) would have induced high toxicity levels.

In order to study the impact of the isopropyl, as well as the PUFA moieties, for anti-carbonyl stress activity, commercial resveratrol (Rcom), R-5OiP (13), R-4'LA and R-4'DHA [42,43] were evaluated and compared to the new alkyl lipophenols 15a and 15b (Fig. 5A). Derivatives bearing both alkyl and PUFA substituents, presented the best protective effects under carbonyl stress: $29 \%$ and $35 \%$ increase in viability using $80 \mu \mathrm{M}$ of R-5OiP-4'LA (15a) and R-5OiP-4'DHA (15b), respectively. By contrast, no significant cell protection was observed for the four other derivatives (R-com, R-5OiP (13), R-4'LA and R-4'DHA), whose chemical structures presented isopropyl, or PUFA substituent, or no additional substituent. These interesting results confirm the importance of the $O$-isopropyl-resorcinol group, as well as the necessity of a PUFA substituent for the anti-carbonyl stress activity, as was observed for the phloroglucinol LEAD series [23]. In agreement with the toxicity results, the protective effect of the esterified compounds (15a and 15b) and the loss in cell viability observed using R-5OiP (13), confirm the stability of the ester bound of these lipophenols during the cell protection assay.

The anti-carbonyl potential of resveratrol has been described by Wang et al. who demonstrated the formation of an adduct by coincubation of resveratrol with acrolein at equimolar concentration [18], and by Shen et al. with methyl glyoxal [45]. Resveratrol was able to scavenge toxic aldehydes and formed a heterocyclic ring at the C-2 and $\mathrm{C}-3$ positions through nucleophilic addition. Resveratrol protection of acrolein-treated cells was also attributed to a direct stimulatory action on mitochondrial bioenergetics [46]. Here, in our cellular assay, natural resveratrol did not display sufficient anti-carbonyl stress activity to counteract toxic effects of high doses of atRAL. However, increasing the nucleophilicity of resorcinol backbone with an isopropyl group, and increasing lipophilicity of this polyphenol by addition of a PUFA, prevented aldehyde toxicity.

Regarding the antioxidant profiles of resveratrol derivatives, shown in Fig. 5B, $40 \mu \mathrm{M}$ of commercial resveratrol (R-com) was able to reach the oxidative status of cells that did not received the oxidant stressor $\mathrm{H}_{2} \mathrm{O}_{2}$. Logically, the introduction of a fatty acid moiety (LA or DHA) and/or an alkyl residue masking the phenolic function, led to a loss of antioxidant capacity compared to R-com. However, both the studied alkyl-lipophenols R-5OiP-4'LA (15a) and R-5OiP-4'DHA (15b) were still potent antioxidant derivatives, as, at $80 \mu \mathrm{M}$, they decreased ROS production by up to $47 \%$ and $37 \%$, respectively. The reduction of the accessibility of two phenolic functions on the resveratrol backbone still allowed $\mathbf{1 5 a}$ and $\mathbf{1 5 b}$ to be potent antioxidants able to reduce oxidative status in a dose-dependent manner.

Thus, both resveratrol alkyl-lipophenols 15a and 15b exhibited an interesting protection against the double COS in ARPE-19 cells.

\subsection{Synthesis and anti-COS evaluation of flavonoid lipophenol analogues}

Flavonoids are widely known as potent antioxidants by direct or indirect action $[13,47,48]$. We worked on two natural flavonoid derivatives, quercetin and catechin (Fig. 1).

Quercetin (3,3', $4^{\prime}, 5,7$-pentahydroxyflavone, Q) is a plant flavonol, which is present in various quantities in many fruit and vegetables, including apple, cranberry, red onion, asparagus, spinach, walnuts and coriander. Over the past decades, quercetin has gained research interest due to its numerous pharmacological activities. In addition to its antioxidant properties, quercetin was proven to have anti-inflammatory, antidiabetic, anticancer, cardiovascular, hepato-protective, antiplatelet, antibacterial and neuroprotective properties [49]. Regarding its antioxidant action, quercetin acts by scavenging free radicals, and also increases antioxidant enzymes, such as glutathione peroxidase, superoxide dismutase and catalase [50]. (+)-Catechin (C) is a flavanol abundant in berries, chocolate, cacao and green tea that makes a significant contribution to total dietary antioxidant intake. Catechin is the monomer of proanthocyanidin B2, whose protection against A2E photo-oxidation-induced apoptosis has been shown in ARPE-19 cells [35]. Compared to quercetin, catechin should have increased nucleophilic properties coming from the A ring, due to the absence of the carbonyl function link to this aromatic cycle.

Distinctive chemical structures related with flavonoid antioxidant activities have been established including hydroxyl groups of the A-ring (resorcinol), ortho-dihydroxy arrangement in the B-ring (catechol), and in the case of quercetin, C2-C3 unsaturated bond combined with C-4 carbonyl group in the C-ring [48]. Moreover, the position instead of total number of hydroxyl groups, considerably influences the efficiency of antioxidant activity [51]. The B-ring hydroxyl structure is the utmost significant actor of scavenging oxygen free radicals [52]. In addition, according to the study of Hong et al. [53], radical scavenging properties of catechin seem to be less affected by acylation at the 3 or 7 position compared to acylation of the catechol moiety. Thereby, in order to conserve the best antioxidant properties, the B-ring, corresponding to the catechol moiety, was left free of any substituent, and position 3 was selected to introduce the PUFA moiety on both catechin and quercetin. Finally, to preserve the alkylated resorcinol group needed for the anti-carbonyl stress activity in both phloroglucinol and resveratrol

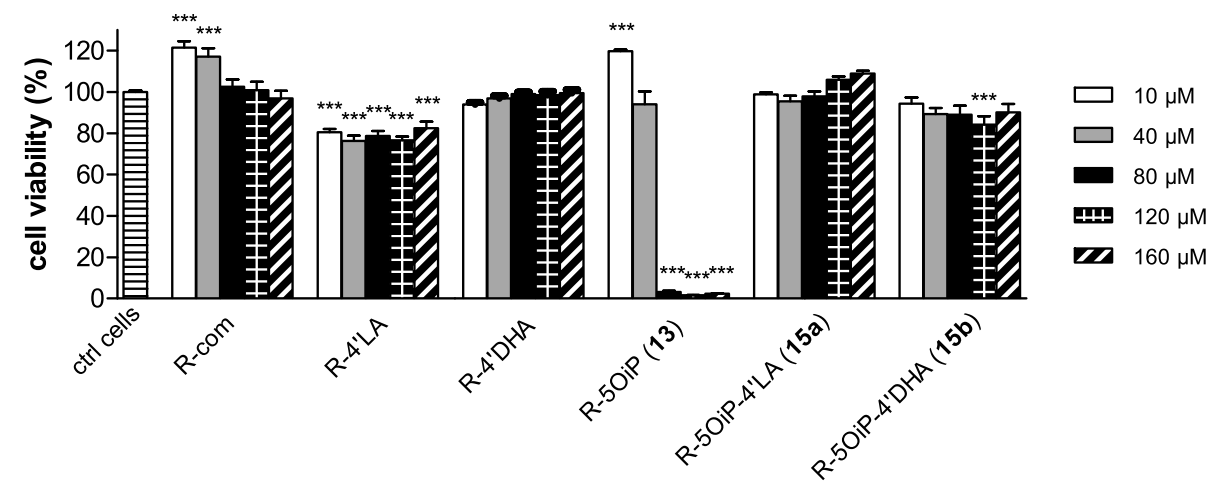

Fig. 4. Evaluation of cytotoxicity of resveratrol derivatives; natural resveratrol (R-com), R-4'LA [42], R-4'DHA [43], R-5OiP (13), R-5OiP-4'LA (15a) and R-5OiP-4'DHA (15b). Results are expressed in mean \pm SEM and are from $n=3-5$ independent experiments. ARPE-19 cell viability (MTT) after incubation of resveratrol derivatives $(0-160 \mu \mathrm{M})$. The data are expressed as the percentage of non-treated control cells. ${ }^{*} \mathrm{p}<0.05,{ }^{* *} \mathrm{p}<0.01,{ }^{* * *} \mathrm{p}<0.001$, versus non-treated control cells. 
(A)

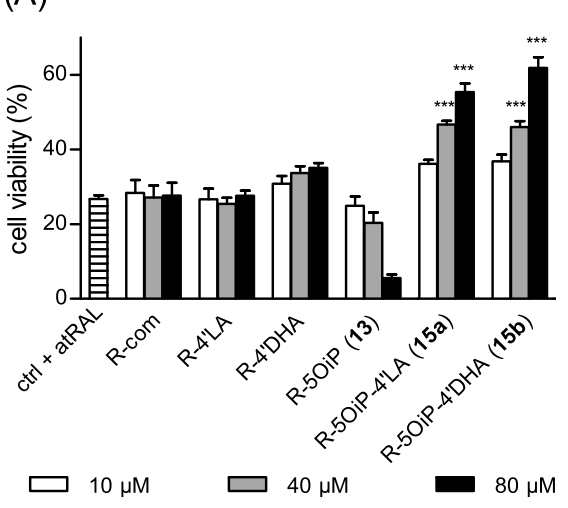

(B)

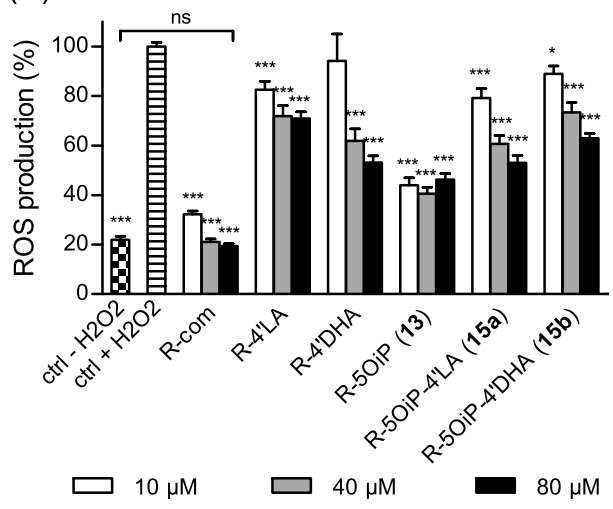

Fig. 5. In vitro anti-COS evaluation of resveratrol derivatives. Comparison of natural resveratrol (Rcom), R-4'LA [42], R-4'DHA [43], R-5OiP (13), R-5OiP-4'LA (15a) and R-5OiP-4'DHA (15b) activities. Results are expressed in mean \pm SEM and are from $\mathrm{n}=3-5$ independent experiments. (A) Anti-carbonyl stress assay: ARPE-19 cell viability (MTT) after incubation of resveratrol derivatives $(0-80 \mu \mathrm{M})$ and atRAL $(15 \mu \mathrm{M})$. The data are expressed as the percentage of non-treated and non-exposed to atRAL control cells. ${ }^{*} \mathrm{p}<0.05, * * \mathrm{p}<0.01$, ***p $<$ 0.001 , versus non-treated and exposed to atRAL cells. (B) Antioxidant assay: representation of ROS production (DCFDA probe) after incubation of resveratrol derivatives $(0-80 \mu \mathrm{M})$ and $\mathrm{H}_{2} \mathrm{O}_{2}(600 \mu \mathrm{M})$ in ARPE-19 cells. The data are expressed as the percentage of non-treated and exposed to $\mathrm{H}_{2} \mathrm{O}_{2}$ cells. *p $<0.05,{ }^{* *} \mathrm{p}<0.01, * * * \mathrm{p}<0.001$, versus non-treated and exposed to $\mathrm{H}_{2} \mathrm{O}_{2}$ cells. series, two positions of the A-ring, that mimic the resorcinol moiety of the LEADs A/B, were selected to introduce the isopropyl; both positions 5 and 7 were alkylated in order to evaluate the most favorable for carbonyl stress protection. As for resveratrol derivatives, lipophilic catechin and quercetin bearing only the PUFA moiety (C-3PUFA and Q-3PUFA) [42], and alkyl-flavonoids bearing only the isopropyl moiety (C-5OiP 18b, C-7OiP 18a, Q-5OiP 23 and Q-7OiP 35), were produced to evaluate their impact on biological properties.

\subsubsection{Catechin lipophenols synthesis and evaluation}

Synthesis of alkyl-catechin derivatives C-7OiP (18a) and C-5OiP (18b), as well as the alkyl-lipophenol derivatives C-3LA-7OiP (19a) and C-3LA-5OiP (19b), are presented in Scheme 3.

The first step of the synthesis was the protection of the catechol moiety of catechin by a diphenyldioxole in acetonitrile [54]. The alkylation step allowed access to both isomers $\mathbf{1 7 a}$ and $\mathbf{1 7} \mathbf{b}$ with $1 / 1$ proportion in $37 \%$ yield. The two isomers were separated by column chromatography and engaged separately for the end of the synthesis. Deprotection of the catechol was performed by hydrogenation using palladium hydroxide and led to the desired alkylated catechin derivatives: C-7OiP (compound 18a) and C-5OiP (compound 18b). The lipophenols were then obtained using TFA and freshly prepared linoleyl chloride, as described by Uesato et al. [55]. Compounds C-3LA-7OiP (19a) and C-3LA-5OiP (19b) were isolated in sufficient quantities to be evaluated in vitro.

Commercial catechin (C-com), as well as the two alkylated catechins C-5OiP (18b) and C-7OiP (18a), displayed no toxicity on ARPE-19 cells
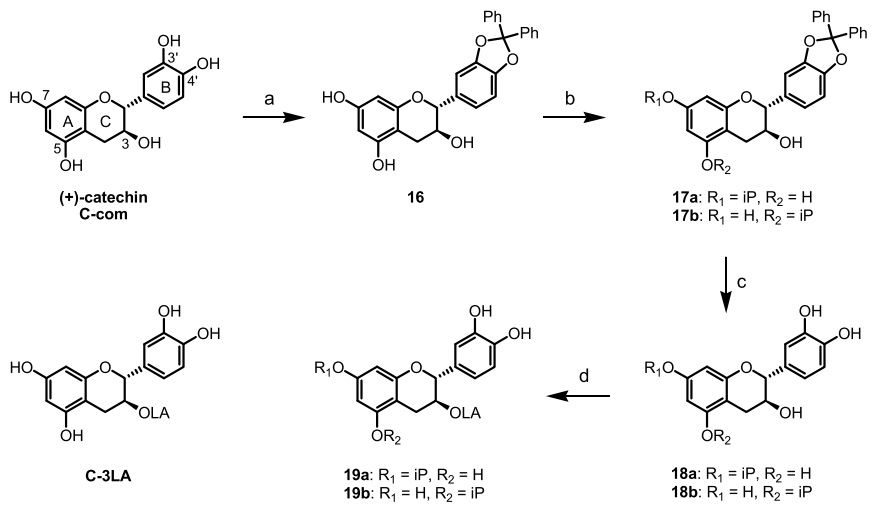

18a: $R_{1}=i P, R_{2}=H$

Scheme 3. Synthesis and chemical structures of (+)-catechin derivatives. Reagents and conditions: (a) $\mathrm{Ph}_{2} \mathrm{CCl}_{2}, \mathrm{~K}_{2} \mathrm{CO}_{3}, \mathrm{MeCN}, \mathrm{rt}, 23 \mathrm{~h}, 33 \%$; (b) diisopropylsulfate, $\mathrm{K}_{2} \mathrm{CO}_{3}$, acetone, $40{ }^{\circ} \mathrm{C}, 70 \mathrm{~h}, 37 \%$ separable mixture of $17 \mathrm{a}$ (19\%) and 17b (18\%); (c) $\mathrm{H}_{2}, \mathrm{Pd}(\mathrm{OH})_{2}$, THF/EtOH, rt, 18a : $42 \mathrm{~h}, 56 \%$ and 18b : 64 h, 72\%; (d) LACOCl, TFA, 1,4-dioxan, rt, 68 h, 1.5\% for 19a and 19b. up to $160 \mu \mathrm{M}$ (Fig. 6). However, C-3LA [42] and C-3LA-5OiP (19b) displayed an important cell death above $120 \mu \mathrm{M}$. Toxicity was even higher for C-3LA-7OiP (19a) with no observed cell viability at $80 \mu \mathrm{M}$. In contrast with resveratrol derivatives, alkylated catechins 18a and 18b displayed no toxicity, but association of PUFA and alkyl on catechin backbone (19a and 19b), resulted in high toxicity in the ARPE-19 cell line.

Interestingly, despite the absence of cell protection using C-com, C3LA was able to display a mild protection against carbonyl stressor, with dose-dependent effects. The catechin ring, in contrast to resveratrol or phloroglucinol ring, should be nucleophilic enough to show a protective effect against carbonyl stressor, without the presence of isopropyl function on the resorcinol moiety (responsible for inductive effect). The direct trapping of RCS, such as methylglyoxal (an intermediate reactive carbonyl of AGE formation) by natural catechin was described in cellfree experiments by Peng et al. [56]. Wang confirmed this observation in 2010, by identifying adducts produced by co-incubation of catechin with glyoxal, methylglyoxal and acrolein [32]. Zhu et al. also demonstrated trapping of lipid-derived $\alpha, \beta$-unsaturated aldehydes, which have been implicated as causative agents in the development of carbonyl stress-associated pathologies (i.e. 4-HNE and acrolein), by several (poly) phenols including catechin [31]. However, most of those adducts have been observed only under simulated physiological conditions but rarely in actual cellular media. As expected, in our work, the addition of isopropyl, preferentially at the 7 position (C-3LA-7OiP (19a), Fig. 7A), increased cellular protection when tested at $40 \mu \mathrm{M}$ (below the toxic concentration of 19a). The position of the isopropyl group seemed to influence anti-carbonyl stress activity, as well as the cytotoxic profile of the derivatives. Alkylation at the 7 position favor aldehyde trapping in both C8 and C6 positions, whereas alkylation at the 5 position orients the formation of adduct only with C6. Both sites were reported in the literature to react with aldehyde, however, some work performed on the activity of epigallocatechin-3-O-gallate (EGCG) led only to the identification of the C8 adduct [57]. Unexpectedly, the most active lipophenol against carbonyl stress in this series, C-3LA-7OiP (19a), was also the most toxic compound.

As expected, C-com, and the two alkylated derivatives C-5OiP (18b) and C-7OiP (18a), displayed the best antioxidant activity with equivalent ROS production levels compared to control cells non-exposed to $\mathrm{H}_{2} \mathrm{O}_{2}$, as shown in Fig. 7B. With two substituents reducing free phenolic functions, C-3LA-5OiP (19b) and C-3LA-7OiP (19a) displayed dosedependent effects on ROS produced by $\mathrm{H}_{2} \mathrm{O}_{2}$ treatment, with a decrease by up to $60 \%$ and $67 \%$ in ROS production, respectively, at 80 $\mu \mathrm{M}$.

Despite these interesting antioxidant properties, C-3LA-5OiP (19b) lacked potent anti-carbonyl stress activity (only $10 \%$ increase in viability observed at $80 \mu \mathrm{M}$ ) and C-3LA-7OiP (19a) showed high toxicity 


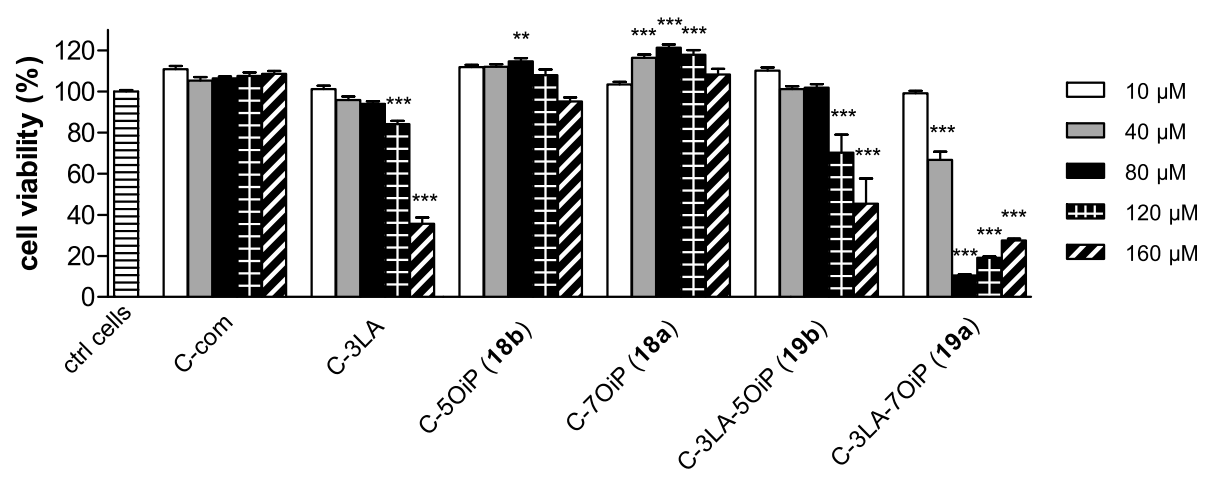

Fig. 6. Evaluation of cytotoxicity of (+)-catechin derivatives; natural (+)-catechin (C-com), C-3LA [42], C-5OiP (18b), C-7OiP (18a), C-3LA-5OiP (19b) and C-3LA-7OiP (19a). Results are expressed in mean \pm SEM and are from $n=3-5$ independent experiments. ARPE-19 cell viability (MTT) after incubation of catechin derivatives $(0-160 \mu \mathrm{M})$. The data are expressed as the percentage of non-treated control cells. ${ }^{*} \mathrm{p}<0.05$, $* * \mathrm{p}<0.01$, ***p $<0.001$, versus non-treated control cells.

(A)

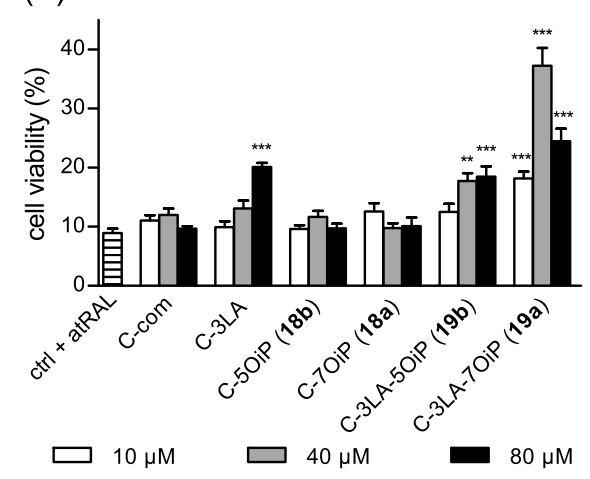

(B)

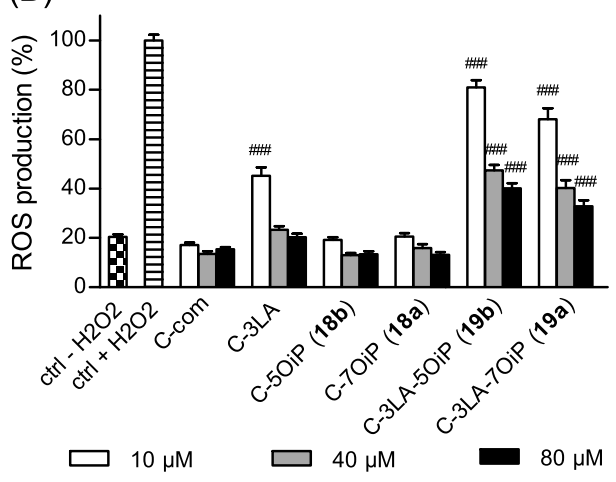

Fig. 7. In vitro anti-COS evaluation of $(+)$-catechin derivatives. Comparison of natural $(+)$-catechin (Ccom), C-3LA [42], C-5OiP (18b), C-7OiP (18a), C-3LA-5OiP (19b) and C-3LA-7OiP (19a) activities. Results are expressed in mean \pm SEM and are from $n$ $=3-5$ independent experiments. (A) Anti-carbonyl stress assay: ARPE-19 cell viability (MTT) after incubation of catechin derivatives $(0-80 \mu \mathrm{M})$ and atRAL $(15 \mu \mathrm{M})$. The data are expressed as the percentage of non-treated and non-exposed to atRAL control cells. ${ }^{*} \mathrm{p}<0.05, * * \mathrm{p}<0.01,{ }^{* * *} \mathrm{p}<0.001$, versus non-treated and exposed to atRAL cells. (B) Antioxidant assay: representation of ROS production (DCFDA probe) after incubation of catechin derivatives (0-80 $\mu \mathrm{M})$ and $\mathrm{H}_{2} \mathrm{O}_{2}(600 \mu \mathrm{M})$ in ARPE-19 cells. The data are expressed as the percentage of non-treated and exposed to $\mathrm{H}_{2} \mathrm{O}_{2}$ cells. All conditions have a p-value $<$ 0.001 versus non-treated and exposed to $\mathrm{H}_{2} \mathrm{O}_{2}$ control cells. \#p $<0.05, \# \# \mathrm{p}<0.01$, \#\#\#p $<0.001$, versus non-treated and non-exposed to $\mathrm{H}_{2} \mathrm{O}_{2}$ cells.

(no survival from $80 \mu \mathrm{M}$ ), making these derivatives less interesting antiCOS candidates compared to the resveratrol series.

\subsubsection{Quercetin-5OiP lipophenols synthesis and evaluation}

Lipophilic quercetins Q-3LA and Q-3DHA were produced as previously described [42] and two original pathways were developed to access the lipophenol derivatives of quercetin-50iP (29) and quercetin-7OiP (39a/b) (Schemes 4 and 5).

A first chemical strategy was developed in order to access the lipophenol derivative Q-3LA-5OiP (29) and the PUFA-free analogue Q5OiP (23) (Scheme 4).

In order to access quercetin-5OiP derivatives, we started from commercially available rutin using the diholoside rutinoside as protecting group for the phenolic function at the 3 position. The first step was the protection of the phenols in positions $7,3^{\prime}$ and $4^{\prime}$ with benzyl groups. Alkylation of the phenol in position 5 was performed with 2-bromopropane by heating at $80^{\circ} \mathrm{C}$ in dry DMF $(88 \%)$. Cleavage of rutinoside in acidic conditions gave compound 22, which was either debenzylated to access desired Q-5OiP (23), or acetylated in position 3 to pursue the lipophenol synthesis. Benzyl groups were removed by hydrogenation to be replaced by silyl protecting groups, which can be easily deprotected without damaging PUFA moiety leading to compound 26. Acetate in position 3 was then cleaved using ammonia solution in $\mathrm{MeOH}$ at $0{ }^{\circ} \mathrm{C}$ to allow esterification with fatty acid. Final deprotection of TIPS group gave the desired Q-3LA-5OiP (29).

For comparison purposes, commercial quercetin (Q-com) and Q-3LA [42] properties were also evaluated in vitro. Under the conditions tested, Q-com was found to be toxic for the ARPE-19 cell line from $80 \mu \mathrm{M}$
(Fig. 8). Introduction of the isopropyl moiety (Q-5OiP, 23) reduced the toxicity with mild mortality starting at $160 \mu \mathrm{M}$. A similar reduction was observed with PUFA introduction, as Q-3LA displayed no toxicity up to $160 \mu \mathrm{M}$, and was found to increase cell viability (156\% cell viability at $80 \mu \mathrm{M})$. However, the alkyl-lipophenol derivative Q-3LA-5OiP (29) was very toxic, with no survival at $80 \mu \mathrm{M}$.

Regarding anti-carbonyl stress properties, here again the presence of isopropyl, as well as the PUFA moiety, seemed decisive for cell protection. As shown in Fig. 9A, Q-com, Q-3LA and Q-5OiP (23) did not display any protective effect against atRAL toxicity, whereas Q-3LA-5OiP (29) protected cells with $50 \%$ increase in cell survival at $80 \mu \mathrm{M}$. Even if toxic at $80 \mu \mathrm{M}$, a high anti-carbonyl stress activity was observed at this concentration and can be explained by the differences between cytotoxicity and anti-carbonyl stress activity protocols (different incubation times).

Finally, antioxidant capacity was evaluated and is reported in Fig. 9B. The di-substituted lipophenol derivative Q-3LA-5OiP (29) was efficient to reduce $\mathrm{H}_{2} \mathrm{O}_{2}$-induced ROS production by $53 \%$ at $80 \mu \mathrm{M}$. Here again, a dose-dependent antioxidant potency was still observable despite the reduction of two free phenolic functions of quercetin. A similar protection profile was observed for the isopropyl-free analogue Q-3LA, which suggests that position 5 is not primordial for ROS scavenging activity. This was also confirmed by comparing Q-com and Q5OiP (23) cell treatment, which led to low ROS levels.

To conclude, in vitro assessments indicate that in this series, alkylated resorcinol and PUFA moieties are necessary for anti-carbonyl stress activity, as observed for the phloroglucinol [23] and resveratrol series. Derivative Q-3LA-5OiP (29) displays interesting anti-COS properties, however, its high cytotoxicity on ARPE-19 has to be considered for 


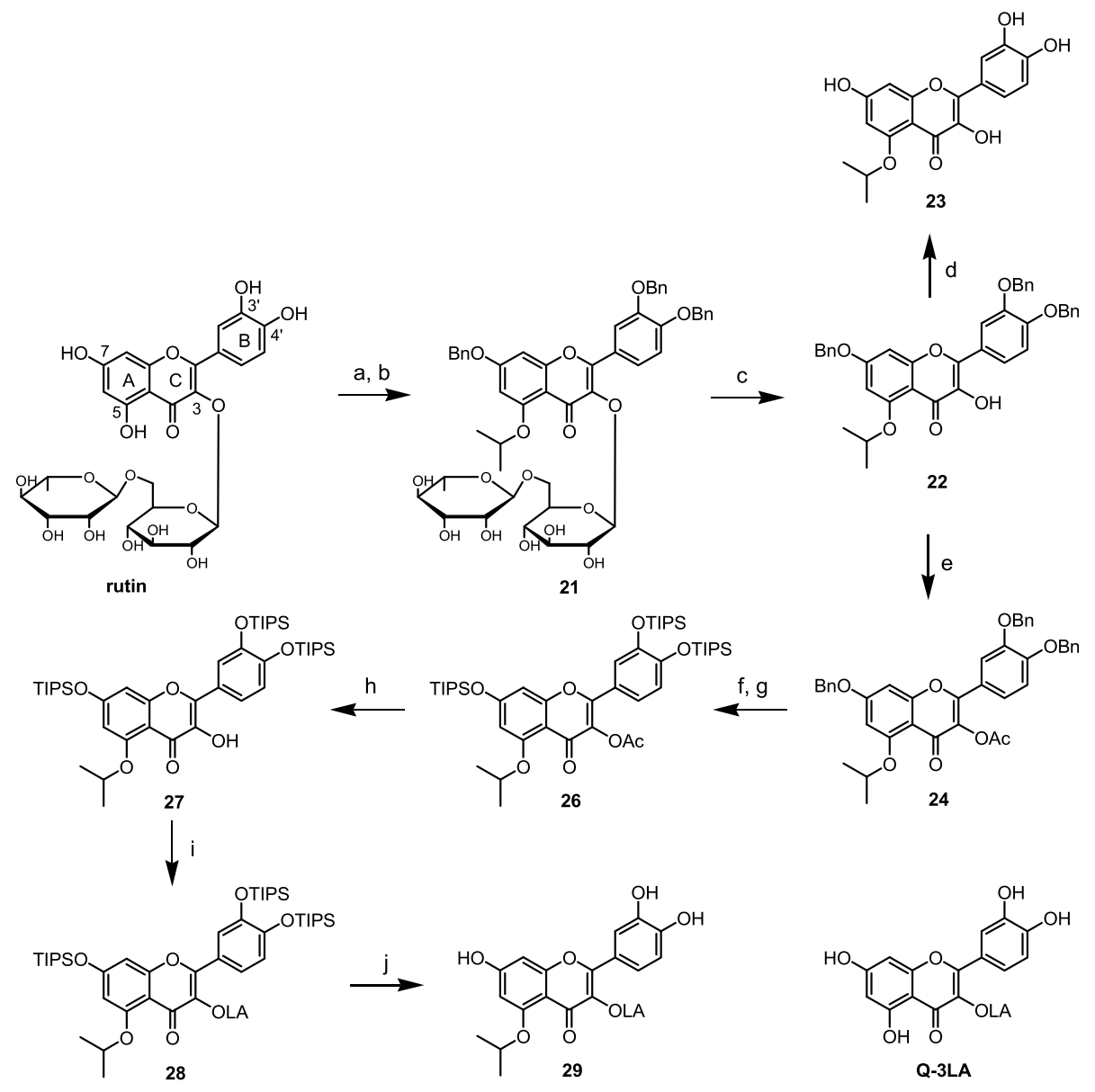

Scheme 4. Synthesis and chemical structures of quercetin-5OiP derivatives. Reagents and conditions: (a) $\mathrm{BnBr}, \mathrm{K}_{2} \mathrm{CO}_{3}, \mathrm{DMF}, 40{ }^{\circ} \mathrm{C}, 4 \mathrm{~h}, 61 \%$; (b) 2 -bromopropane, $\mathrm{K}_{2} \mathrm{CO}_{3}$, DMF, $80^{\circ} \mathrm{C}, 18 \mathrm{~h}, 88 \%$; (c) $\mathrm{HCl}$, EtOH, $70^{\circ} \mathrm{C}, 5 \mathrm{~h}, 98 \%$; (d) $\mathrm{H}_{2}$, Pd/C, THF/EtOH, rt, 21 h, 69\%; (e) pyridine, Ac 2 O, rt, 20 h, 100\%; (f) $\mathrm{H}_{2}$, Pd/C, THF/EtOH, rt, 15 h, 100\%; (g) TIPS-OTf, NEt 3 , THF, rt, 4 h, 62\%; (h) $\mathrm{NH}_{3} / \mathrm{MeOH}$, DCM, $0{ }^{\circ} \mathrm{C}, 3$ h, 88\%; (i) LA, DCC, DMAP, DCM, rt, 23 h, 54\%; (j) NEt $3 / 3 \mathrm{HF}, \mathrm{THF}^{2}, \mathrm{rt}, 15 \mathrm{~min}, 72 \%$.

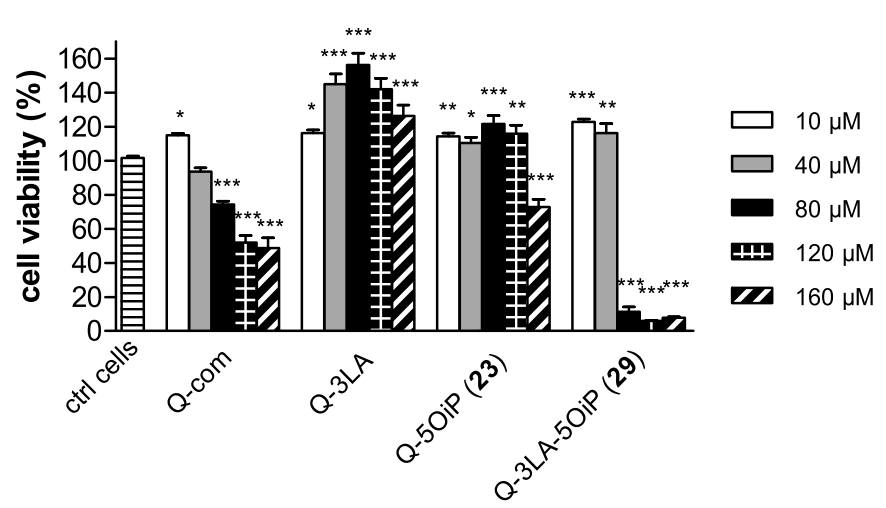

Fig. 8. Evaluation of cytotoxicity of quercetin-50iP derivatives; natural quercetin (Q-com), Q-3LA [42], Q-5OiP (23) and Q-3LA-5OiP (29). Results are expressed in mean \pm SEM and are from at $\mathrm{n}=3-5$ independent experiments. ARPE-19 cell viability (MTT) after incubation of quercetin-5OiP derivatives $(0-160 \mu \mathrm{M})$. The data are expressed as the percentage of non-treated control cells. ${ }^{*} \mathrm{p}<0.05,{ }^{* *} \mathrm{p}<0.01,{ }^{* * *} \mathrm{p}<0.001$, versus non-treated control cells.

further evaluations.

\subsubsection{Quercetin-7OiP lipophenols synthesis and evaluation}

A second chemical strategy, presented in Scheme 5, was developed for the synthesis of alkyl quercetin Q-7OiP (35) and the two lipophenol derivatives Q-3LA-7OiP (39a) and Q-3DHA-7OiP (39b).

For the synthesis of Q-7OiP derivatives, the catechol of the commercial quercetin was first protected by diphenyldioxole, followed by the protection of the remaining phenolic functions with acetate moieties. Selective deprotection of phenol in position 7 was then performed according to $\mathrm{Li}$ et al. using thiophenol and imidazole in $\mathrm{N}$ methylmorpholine in quantitative yield [58]. Compound $\mathbf{3 2}$ was then alkylated with diisopropylsulfate leading to derivative 33 in $91 \%$ yield. Hydrogenation of diphenyldioxole with palladium hydroxide led to compound 34, which can be either deacetylated to access desired Q-7OiP (35) or protected with silyl ethers to access protected derivative 36. Deacetylation of $\mathbf{3 6}$ with diluted ammonia solution in $\mathrm{MeOH}$ at $0{ }^{\circ} \mathrm{C}$ allowed access to compound 37, which could be selectively esterified in position 3 by PUFA ( $86 \%$ for LA and $85 \%$ for DHA derivatives). Resulting compounds underwent a final TIPS deprotection leading to the desired lipophenol derivatives Q-3LA-7OiP (39a) and Q-3DHA-7OiP (39b).

Toxicity and activity of quercetin-7OiP lipophenols Q-3LA-7OiP (39a) and Q-3DHA-7OiP (39b), were compared to Q-com, Q-3LA [42], Q-3DHA [42] and Q-7OiP (35). Toxicity profile (Fig. 10) shows that Q-7OiP (35) was highly toxic even at low concentrations with no survival observed at $40 \mu \mathrm{M}$. The position of the alkyl moiety appeared critical, as Q-5OiP (23, Fig. 8) did not display such high toxicity. Reduction of Q-com toxicity was observed by introduction of the PUFA moieties, as Q-3DHA and Q-3LA increased ARPE-19 cells viability from 10 to $80 \mu \mathrm{M}$ until toxicity appeared. Alkyl-lipophenol derivatives Q-3LA-7OiP (39a) and Q-3DHA-7OiP (39b) did not display any toxicity up to $160 \mu \mathrm{M}$, and Q-3LA-7OiP (39a) even increased cell survival at 160 $\mu \mathrm{M}$ (156\% cell viability).

As also observed for the resveratrol and catechin series, in our 
(A)

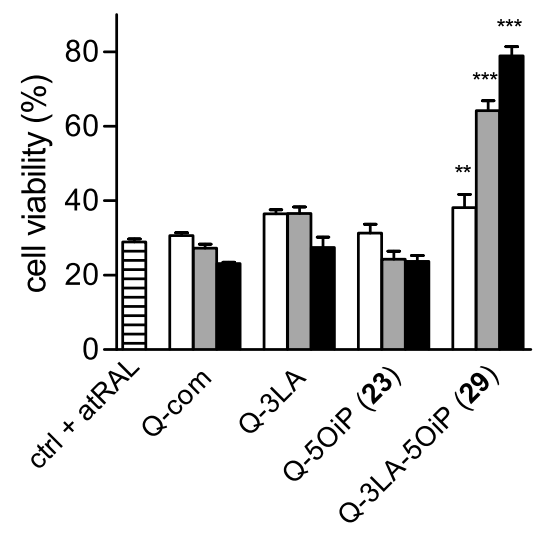

$10 \mu \mathrm{M} \square 40 \mu \mathrm{M} \square 80 \mu \mathrm{M}$
(B)

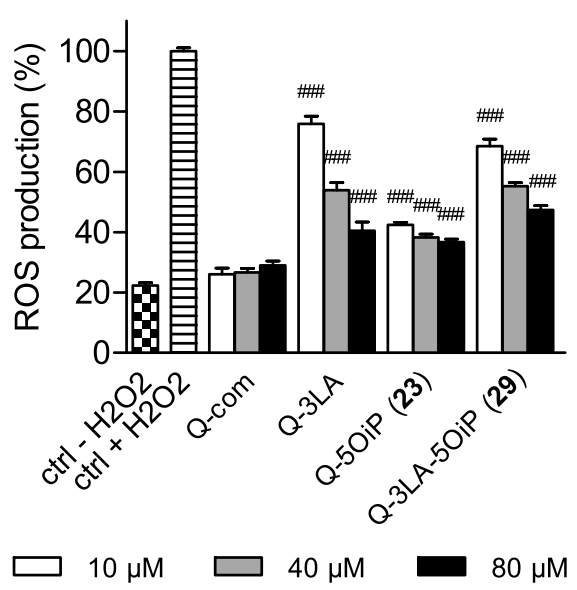

Fig. 9. In vitro anti-COS evaluation of quercetin-5OiP derivatives. Comparison of natural quercetin (Q-com), Q-3LA [42], Q-5OiP (23) and Q-3LA-5OiP (29) activities. Results are expressed as mean \pm SEM and are from $\mathrm{n}=3-5$ independent experiments. (A) Anti-carbonyl stress assay: ARPE-19 cell viability (MTT) after incubation of quercetin-5OiP derivatives $(0-80 \mu \mathrm{M})$ and atRAL $(15 \mu \mathrm{M})$. The data are expressed as the percentage of non-treated and non-exposed to atRAL control cells. *p $<0.05, * * \mathrm{p}<0.01,{ }^{* * *} \mathrm{p}<$ 0.001 , versus non-treated and exposed to atRAL cells. (B) Antioxidant assay: representation of ROS production (DCFDA probe) after incubation of quercetin-5OiP derivatives $(0-80 \mu \mathrm{M})$ and $\mathrm{H}_{2} \mathrm{O}_{2}(600$ $\mu \mathrm{M})$ in ARPE-19 cells. The data are expressed as the percentage of non-treated and exposed to $\mathrm{H}_{2} \mathrm{O}_{2}$ cells. All conditions have a p-value $<0.001$ versus non-treated and exposed to $\mathrm{H}_{2} \mathrm{O}_{2}$ control cells. \#p $<$ $0.05, \# \# \mathrm{p}<0.01, \# \# \# \mathrm{p}<0.001$, versus non-treated and non-exposed to $\mathrm{H}_{2} \mathrm{O}_{2}$ cells.

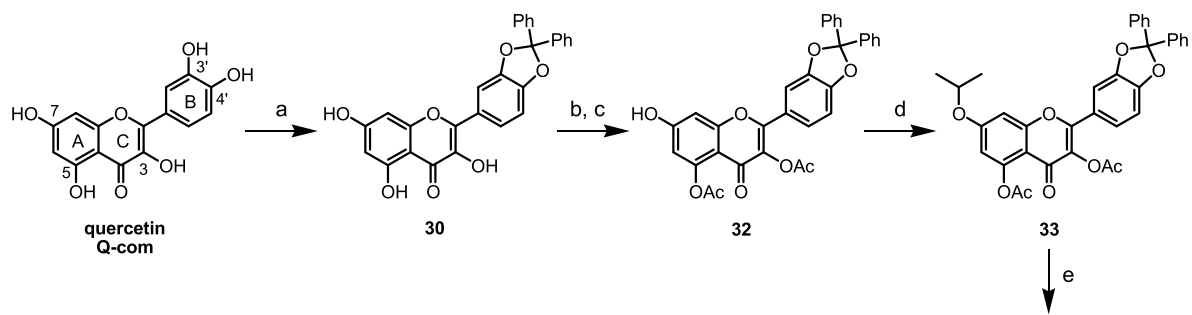

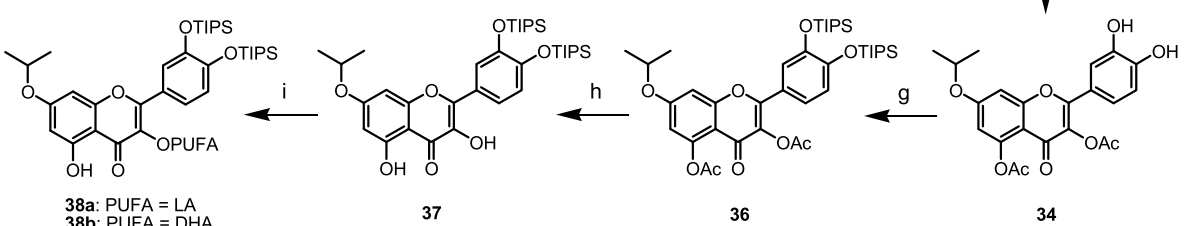<smiles>CC(C)Oc1cc(O)c2c(=O)c(OC(C)C)c(-c3ccc(O)c(O)c3)oc2c1</smiles>
39a: PUFA $=$ LA
37<smiles></smiles>

Q-3DHA<smiles>CC(C)Oc1cc(O)c2c(=O)c(O)c(-c3ccc(O)c(O)c3)oc2c1</smiles>

35

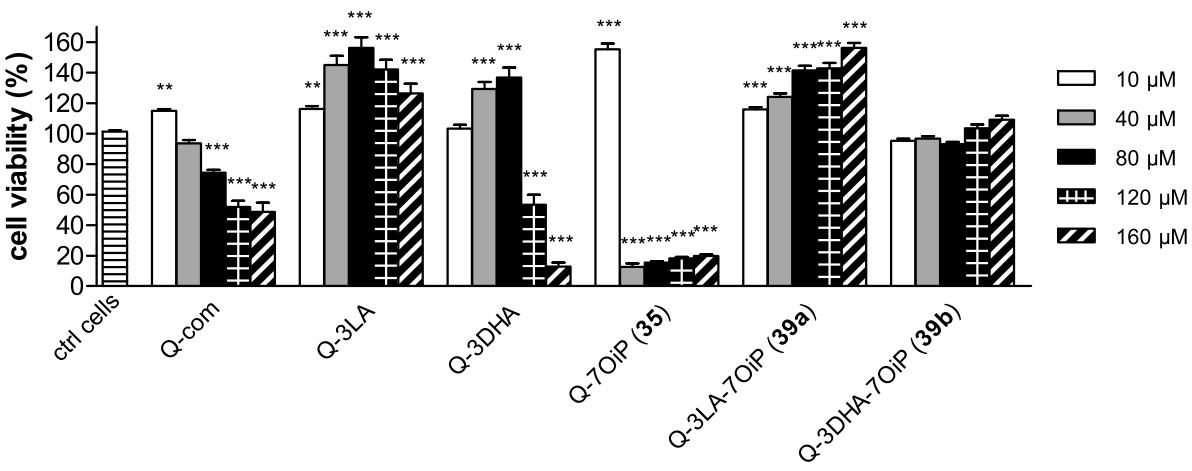

Scheme 5. Synthesis and chemical structures of quercetin-7OiP derivatives. Reagents and conditions: (a) $\mathrm{Ph}_{2} \mathrm{CCl}_{2}, \mathrm{Ph}_{2} \mathrm{O}, 175{ }^{\circ} \mathrm{C}, 2 \mathrm{~h}, 89 \%$; (b) pyridine, $\mathrm{Ac}_{2} \mathrm{O}, \mathrm{rt}, 20 \mathrm{~h}, 100 \%$; (c) $\mathrm{PhSH}$, imidazole, NMP, $0-5{ }^{\circ} \mathrm{C}, 5 \mathrm{~h}, 100 \%$; (d) diisopropylsulfate, $\mathrm{K}_{2} \mathrm{CO}_{3}$, acetone, rt, $22 \mathrm{~h}, 91 \%$; (e) $\mathrm{H}_{2}, \mathrm{Pd}(\mathrm{OH})_{2}$, THF/EtOH, rt, 20 h, 47\%; (f) $\mathrm{NH}_{3} / \mathrm{MeOH}, 0{ }^{\circ} \mathrm{C}, 35 \mathrm{~min}, 46 \%$; (g) TIPS-OTf, $\mathrm{NEt}_{3}$, THF, rt, 10 min, 71\%; (h) $\mathrm{NH}_{3} /$ $\mathrm{MeOH}, \mathrm{DCM}, 0^{\circ} \mathrm{C}, 1 \mathrm{~h}, 100 \%$; (i) PUFA, DCC, DMAP, DCM, rt, 5 h, 38a: $86 \%$ and 38b: 85\%; (j) $\mathrm{NEt}_{3} / 3 \mathrm{HF}$, THF, rt, $30 \mathrm{~min}, 92 \%$ for $39 \mathrm{a}$ and $39 \mathrm{~b}$. cellular assay the natural quercetin alone did not protect against carbonyl stress toxicity (Fig. 11A), whereas it has been reported the formation of adduct by co-incubation of quercetin with several reactive aldehydes (i.e. glyoxal, methylglyoxal and acrolein) in cell-free assays [32]. As expected regarding toxicity profile, the PUFA-free quercetin
Q-7OiP (35) is not protective against carbonyl stress and presents high toxicity. Globally, the addition of the isopropyl on this lipophenol derivatives led to an increase of cell protection against RCS, however, the impact of the isopropyl seemed less important than in the resveratrol, phloroglucinol or quercetin-5OiP lipophenol series. Comparison 
(A)

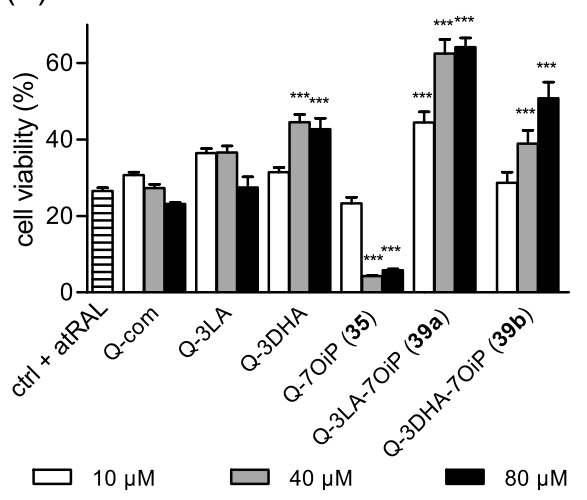

(B)

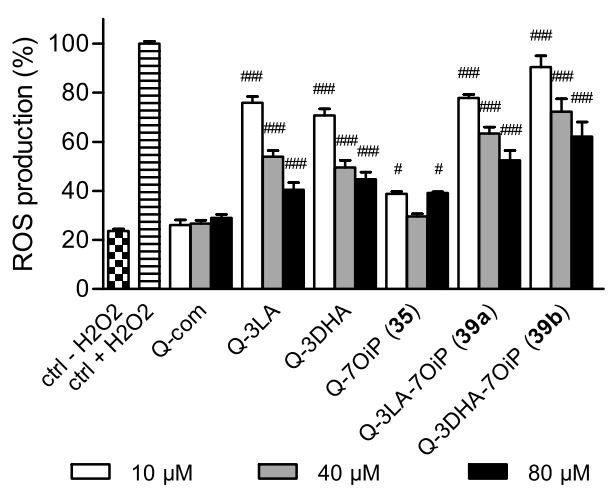

Fig. 11. In vitro anti-COS evaluation of quercetin7OiP derivatives. Comparison of natural quercetin (Q-com), Q-3LA [42], Q-3DHA [42], Q-7OiP (35), Q-3LA-7OiP (39a) and Q-3DHA-7OiP (39b) activities. Results are expressed as mean \pm SEM and are from $n$ $=3-5$ independent experiments. (A) Anti-carbonyl stress assay: ARPE-19 cell viability (MTT) after incubation of quercetin-7OiP derivatives $(0-80 \mu \mathrm{M})$ and atRAL $(15 \mu \mathrm{M})$. The data are expressed as the percentage of non-treated and non-exposed to atRAL control cells. ${ }^{*} \mathrm{p}<0.05,{ }^{* *} \mathrm{p}<0.01,{ }^{*} * * \mathrm{p}<0.001$, versus non-treated and exposed to atRAL cells. (B) Antioxidant assay: representation of ROS production (DCFDA probe) after incubation of quercetin-7OiP derivatives $(0-80 \mu \mathrm{M})$ and $\mathrm{H}_{2} \mathrm{O}_{2}(600 \mu \mathrm{M})$ in ARPE-19 cells. The data are expressed as the percentage of non-treated and exposed to $\mathrm{H}_{2} \mathrm{O}_{2}$ cells. All conditions have a p-value $<0.001$ versus non-treated and exposed to $\mathrm{H}_{2} \mathrm{O}_{2}$ control cells. \#p $<0.05$, \#\#p $<$ 0.01 , \#\#\#p $<0.001$, versus non-treated and non-exposed to $\mathrm{H}_{2} \mathrm{O}_{2}$ cells. between the effect of Q-7OiP (35) and the alkyl-lipophenols Q-3LA-7OiP (39a) and Q-3DHA-7OiP (39b) (increased viability by $38 \%$ and $24 \%$, respectively, at $80 \mu \mathrm{M}$ ) confirmed the importance of the PUFA part to confer high cellular protection against atRAL toxicity using alkyl-(poly) phenol (already observed in other series). Lipid peroxidation of PUFA produces reactive aldehydes (4-HNE or 4-hydroxyhexenal (4-HHE)) [59]. Exposure to excessive 4-HNE or 4-HHE can cause cytotoxicity and is implied in the detrimental pathogenesis of a number of degenerative diseases [60]. However, such lipid peroxidation metabolites are also signaling molecules able to induce gene expression of antioxidant and detoxifying aldehyde enzymes, by activation of the Nrf2 pathway [61]. In ARPE-19, Johansson et al. reported the ability of DHA treatment to induce cellular antioxidant responses, by Nrf2 pathway activation, and to stimulate autophagy [62]. In addition, some works reported also the anti-glycation properties of PUFAs [63,64]. As glycation reaction is caused by reactive aldehydes, anti-glycation properties can be related to anti-carbonyl stress activity. Additional studies reported the cytoprotective effect of PUFAs, by increasing S-phase cell promotion or lipid metabolism [65]. In the retina for example, DHA was shown to protect photoreceptors from oxidative stress by preserving mitochondrial membrane integrity [66]. In most of the (poly)phenol series studied in this work, the introduction of a PUFA part and an alkyl moiety on the (poly)phenol was a prerequisite to provide important cellular protection against atRAL toxicity. This may be due to improved cell penetration due to an increased lipophilicity, and/or a synergic effect of the alkyl-(poly) phenol and the PUFA moiety.

Antioxidant capacities of the derivatives are reported in Fig. 11B. As expected, lower antioxidant profile of the two lipophenols Q-3LA-7OiP (39a) and Q-3DHA-7OiP (39b), compared to natural quercetin, can be explained by introduction of two substituents on phenolic positions. However, Q-3LA-7OiP (39a) and Q-3DHA-7OiP (39b) displayed interesting dose-dependent reduction of ROS close to the alkyl-free lipophenols Q-3DHA and Q-3LA. They decreased by up to $48 \%$ and $38 \%$ of $\mathrm{H}_{2} \mathrm{O}_{2}$ induced ROS production, respectively, when introduced at $80 \mu \mathrm{M}$.

The two di-substituted candidates Q-3LA-7OiP (39a) and Q-3DHA7OiP (39b) are potent anti-COS derivatives for further evaluations, as they displayed interesting dose-dependent protection on ARPE-19 cell line and induced no toxicity up to $160 \mu \mathrm{M}$.

\subsection{Selection of best anti-COS candidates in ARPE-19 cells}

After systematic evaluation of our lipophenol derivatives, only the most promising candidates were selected for further in vitro evaluations. Catechin and quercetin-5OiP lipophenols have been rejected as they display important cytotoxicity in the ARPE-19 cell line. Regarding the close protection provided by either LA or DHA lipophenols, omega-3 DHA lipophenols were selected in view of in vivo evaluation. Indeed, many beneficial effects of DHA have been reported in relation to retinal affections [67], such as anti-inflammatory, neuroprotective effects [28, 68] and antioxidant properties [66]. Visual processing deficits have been improved with DHA supplementation in some clinical studies [25, 67]. Moreover, as predominant PUFA of the photoreceptor membrane, DHA is transported by several specific pathways across the Blood Retina Barrier (BRB) [69]. These properties of DHA are favorable, as increasing the lipophilicity of (poly)phenols using this PUFA, can help the derivatives through retinal cell membranes and across the RPE barrier. In order to select the best candidate for pharmacological development, we compared anti-COS profiles of the most promising lipophenols. According to SAR study of anti-COS assays, selected derivatives bear an isopropyl moiety, as well as DHA lipophilic function.

\subsubsection{Anti-COS comparative assays}

All anti-COS activities of promising alkyl-DHA lipophenols have been summarized at the same concentration $(80 \mu \mathrm{M})$ that displayed the best protective effects, and are represented in Fig. 12. The range of anticarbonyl stress activity is similar for three of the four derivatives: R5OiP-4'DHA (15b) is the only compound that displayed a significantly higher protective effect against atRAL toxicity compared to P-OiP-ODHA (LEAD B), P-OiP-CDHA (8b) or Q-3DHA-7OiP (39b), as shown in Fig. 12A.

The main objective of the (poly)phenol backbone modification was to overcome the low antioxidant activity of P-OiP-ODHA (LEAD B). This goal was achieved, as two of the three new lipophenol derivatives displayed a significant better efficiency against $\mathrm{H}_{2} \mathrm{O}_{2}$-induced ROS production when compared to the LEAD B: 16 and $17 \%$ reduction in ROS production for P-OiP-CDHA (8b) and Q-3DHA-7OiP (39b), respectively, as shown in Fig. 12B. No significant difference in antioxidant evaluation was observed between P-OiP-ODHA (LEAD B) and R-5OiP-4'DHA (15b). Lower efficacy of P-OiP-ODHA (LEAD B) and R-5OiP-4'DHA (15b) could be explained by comparing their number of free phenolic positions as those lipophenols only have one free phenolic function to scavenge ROS. Papuc et al. detailed structure-activity relationship of (poly)phenol antioxidant activity and highlighted that not only the number of free phenols is important for radical scavenging activity but also their position [70]. Indeed, flavonoids with a catechol moiety (B-ring) are the most effective radical scavengers due to the degree of stability conferred by the catechol structure participating in electron delocalization and in the chelation of metals involved in ROS generation. 
(A)

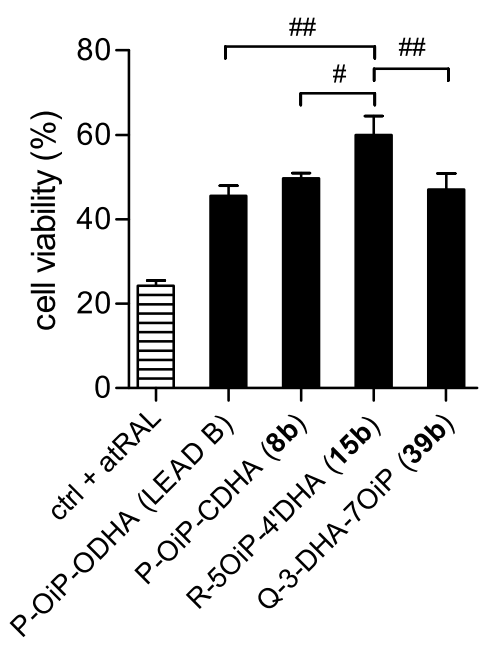

(B)

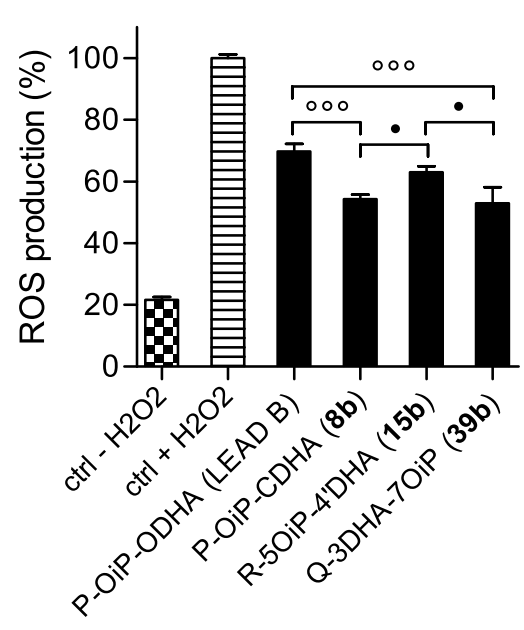

Fig. 12. Comparison of anti-COS activities of selected DHA alkyl-lipophenols: P-OiP-ODHA (LEAD B) [23], P-OiP-CDHA (8b), R-5OiP-4'DHA (15b) and Q-3DHA-7OiP (39b). Results are expressed as mean \pm SEM and are from $n=3-5$ independent experiments. (A) Anti-carbonyl stress assay: ARPE-19 cell viability (MTT) after incubation of lipophenol derivatives $(80 \mu \mathrm{M})$ and atRAL $(15 \mu \mathrm{M})$. The data are expressed as the percentage of non-treated and non-exposed to atRAL control cells. All conditions have a p-value $<0.001$ versus non-treated and exposed to atRAL control cells. \#p $<0.05$, \#\#p $<$ 0.01, \#\#\#p $<0.001$, versus R-5OiP-4'DHA (15b). (B) Antioxidant assay: representation of ROS production (DCFDA probe) after incubation of lipophenol derivatives $(80 \mu \mathrm{M})$ and $\mathrm{H}_{2} \mathrm{O}_{2}(600 \mu \mathrm{M})$ in ARPE-19 cells. The data are expressed as the percentage of non-treated and exposed to $\mathrm{H}_{2} \mathrm{O}_{2}$ cells. All conditions have a p-value $<0.001$ versus non-treated and exposed to $\mathrm{H}_{2} \mathrm{O}_{2}$ control cells and versus non-treated and non-exposed to $\mathrm{H}_{2} \mathrm{O}_{2}$ control cells. ${ }^{\circ} \mathrm{p}<0.05$, ${ }^{\circ} \mathrm{p}<0.01,{ }^{\circ 0} \mathrm{p}<0.001$, versus P-OiP-ODHA (LEAD B). $\bullet \mathrm{p}<0.05, \bullet p<0.01, \bullet \bullet p<0.001$, versus R-5OiP-4'DHA (15b).

\subsubsection{Protection against photo-induced A2E toxicity}

Lipofuscin, a fluorescent lysosomal pigment composed of several lipophilic molecules (bis-retinoids), is associated with age-related pathophysiological processes in the RPE. The best-studied bis-retinoid and the first component of lipofuscin to be identified is A2E. Accumulation and photo-oxidation of the di-retinal conjugate A2E in the RPE through COS mechanisms are known to be one of the critical causes of AMD [5-7,71]. Therefore, the reduction of lipid oxidation is a promising approach to prevent the progression of AMD [72]. RPE cell death was observed by photo-oxidation of A2E due to generation of singlet oxygen and superoxide radicals [73]. A2E can be degraded in epoxide and aldehyde derivatives leading also to carbonyl stress $[7,74]$. This is why we did not only evaluate the protective effects against atRAL toxicity and ROS scavenging properties herein. For an in-depth lipophenol comparison, a more specific cellular assay is the evaluation of the protective effects of DHA alkyl-lipophenols against photo-oxidized A2E toxicity, which more closely resembles AMD cytotoxicity.

Evaluation of survival of the RPE cell line ARPE-19 after incubation of lipophenols with A2E and photo-oxidation by intensive blue light was performed. As represented in Fig. 13, the best protective effect was obtained for Q-3DHA-7OiP (39b) which increased cell viability by $50 \%$ at $80 \mu \mathrm{M}$, whereas P-OiP-ODHA (LEAD B) and P-OiP-CDHA (8b) only improved survival by $16 \%$ and $27 \%$, respectively. R-5OiP-4'DHA (15b) was also an interesting candidate, as it increased cell viability by $43 \%$ at $80 \mu \mathrm{M}$. All DHA-lipophenols are able to protect cells against photo- oxidized A2E toxicity. In order to better compare the four selected alkyl-lipophenols, dose-dependent responses from $10 \mu \mathrm{M}$ to $80 \mu \mathrm{M}$ were performed to calculate Efficiency Concentration $50\left(\mathrm{EC}_{50}\right)$, the concentration of lipophenol needed to protect $50 \%$ of the cells from photooxidized A2E toxicity. P-OiP-ODHA (LEAD B) was less potent than the other derivatives with an $\mathrm{EC}_{50}$ of $145 \mu \mathrm{M}$. This can be explained by its lower ROS scavenging ability. P-OiP-CDHA (8b) was significantly more protective than P-OiP-ODHA (LEAD B) with an $\mathrm{EC}_{50}$ of $64 \mu \mathrm{M}$. This result was in accordance with the anti-COS study, as both lipophenols displayed the same anti-carbonyl stress activity but P-OiP-CDHA (8b) was more efficient at scavenging ROS than its lead P-OiP-ODHA (LEAD B). Surprisingly, R-5OiP-4'DHA (15b) was more potent than P-OiPCDHA (8b), protecting up to $59 \%$ of cells exposed to photo-oxidized A2E at $80 \mu \mathrm{M}\left(\mathrm{EC}_{50}=45 \mu \mathrm{M}\right)$, although it was less efficient at scavenging ROS generated by $\mathrm{H}_{2} \mathrm{O}_{2}$ in the first antioxidant evaluation. However, anti-carbonyl stress activity (higher for R-5OiP-4'DHA (15b) than P-OiPCDHA (8b)) likely participated in the protection of cells against photooxidized A2E. The most efficient lipophenol against A2E toxicity is Q3DHA-7OiP (39b) with an $\mathrm{EC}_{50}$ of $15 \mu \mathrm{M}$ only and a maximal viability of $67 \%$ of cells at $80 \mu \mathrm{M}$. Indeed, this lipophenol, which displayed an equivalent anti-carbonyl stress activity as the others, was the most efficient in scavenging ROS and was therefore the most protective in the assays. Comparison of natural (poly)phenols (i.e. phloroglucinol, resveratrol and quercetin) in the A2E assay showed that quercetin was itself more protective than phloroglucinol and resveratrol against photo-

\begin{tabular}{|c|c|}
\hline Compound & A2E EC $_{\mathbf{5 0}}(\boldsymbol{\mu M})$ \\
\hline P-OiP-ODHA (LEAD B) & $144.60 \pm 28.1$ \\
\hline P-OiP-CDHA (8b) & $63.99 \pm 9.73$ \\
\hline R-5OiP-4'DHA (15b) & $44.87 \pm 6.07$ \\
\hline Q-3DHA-7OiP (39b) & $15.38 \pm 2.53$ \\
\hline
\end{tabular}

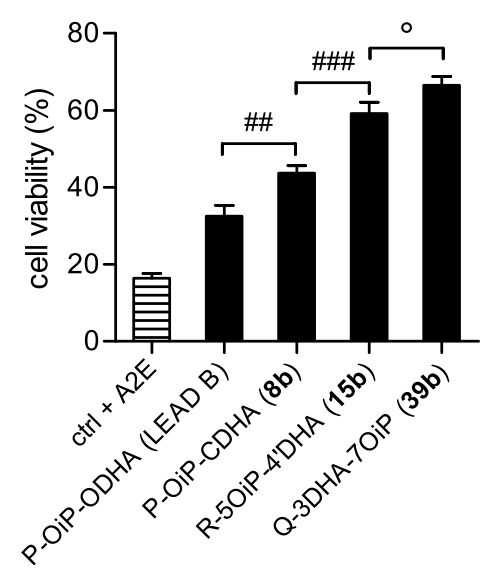

Fig. 13. DHA-lipophenols protection against photooxidized A2E toxicity. Comparison of P-OiP-ODHA (LEAD B) [23], P-OiP-CDHA (8b), R-5OiP-4'DHA (15b) and Q-3DHA-7OiP (39b) protection. Results are expressed as mean \pm SEM and are from $n=3-5$ independent experiments. ARPE-19 cell viability (MTT) after incubation of lipophenol derivatives $(80 \mu \mathrm{M})$ and toxic concentration of photo-oxidized A2E (20 $\mu \mathrm{M}$, blue light exposure $30 \mathrm{~min}$ ). The data are expressed as the percentage of non-treated and non-exposed to $\mathrm{A} 2 \mathrm{E}$ control cells. $\mathrm{EC}_{50}$ are calculated for all lipophenols using dose reponses curves $(0-80$ $\mu \mathrm{M})$ and GraphPad prism software. All conditions have a p-value $<0.001$ versus non-treated and exposed to toxic photo-oxidized A2E control cells. \#p $<0.05$, \#\#p $<0.01$, \#\#\#p $<0.001$, versus P-OiP-CDHA (8b). ${ }^{\circ} \mathrm{p}<0.05,{ }^{\circ 0} \mathrm{p}<0.01,{ }^{\circ 00} \mathrm{p}<$ 0.001 , versus R-5OiP-4'DHA (15b). 
induced toxicity of A2E (data not shown).

Among the three in vitro cell assays performed in this study and the toxicity profile, Q-3DHA-7OiP (39b) has proven to be the most promising anti-COS lipophenol and R-5OiP-4'DHA (15b) an interesting fallback solution.

\subsection{Validation of Q-3DHA-7OiP anti-COS properties in primary rat RPE} cells

Currently, the ARPE-19 cell line and primary cultures are both sources of RPE cells for in vitro models used in fundamental and applied research, including the development of new approaches for ophthalmological disorders [75]. The advantage of cell lines is that they maintain their characteristics over a number of passages and have longer survival times, compared to primary cultures. Moreover, ARPE-19 can be plated at constant cell density throughout the study, whereas primary cultures exhibit more cell density variability [24]. This cell density depends on the cytotoxicity of atRAL and $\mathrm{H}_{2} \mathrm{O}_{2}[24,76]$. Nevertheless, even if ARPE-19 cells are a valuable model for human RPE cells, it seems important to validate the anti-COS effect of the compounds on primary cells to be closer to the physiological protection in vivo [11]. However, the production of primary RPE cells is tedious and time consuming work because it depends on the number of eyeballs available [26,77]. Therefore, only the most promising lipophenol derivative Q-3DHA-7OiP (39b) was evaluated in primary rat RPE cells for its protective action against atRAL toxicity and ROS production. A dose-dependent increase in cell viability was observed for cultures incubated with atRAL and Q-3DHA-7OiP (39b) and is represented in Fig. 14A. Treatment of primary RPE cells with atRAL ( $25 \mu \mathrm{M})$ caused a significant decrease in cell viability ( $26 \%$ of cell viability for untreated control cells), whereas treatment with atRAL and Q-3DHA-7OiP $(40,80$ and $160 \mu \mathrm{M})$ significantly improved cell viability by $15 \%, 22 \%$ and $39 \%$, respectively). Interestingly, the stress condition ( $25 \mu \mathrm{M}$ of atRAL), as well as treatment with up to $80 \mu \mathrm{M}$ Q-3DHA-7OiP, showed very similar effects in primary RPE and ARPE-19 cells, suggesting that both stressor and protector use a common mechanism of action. By contrast, exposure of primary RPE cells to $\mathrm{H}_{2} \mathrm{O}_{2}$ at $600 \mu \mathrm{M}$ for $4 \mathrm{~h}$ increased intracellular ROS levels by 12-fold compared to the untreated cells ( $\mathrm{Ctrl}-\mathrm{H}_{2} \mathrm{O}_{2}$ ). Moreover, cells treated with Q-3DHA-7OiP (40, 80 and $160 \mu \mathrm{M})$ markedly reduced ROS by $81 \%, 91 \%$ and $92 \%$, respectively, in comparison to the non-treated cells, exposed to $\mathrm{H}_{2} \mathrm{O}_{2}$ (Fig. 14B).
The protective effects of Q-3DHA-7OiP (39b) on primary rat RPE cells fully validate the results obtained on ARPE-19 cells. This is particularly true with respect to the carbonyl stress generated by atRAL, as the primary RPE showed a high protection effectiveness of Q-3DHA7OiP. This demonstrates the robustness of the results obtained on the cell line and suggests that these data can be more easily extrapolated to in vivo assays. Moreover, Q-3DHA-7OiP (39b) is now a lead for future investigations. The antioxidant activity is itself remarkable because it is much more effective in the primary RPE compared to ARPE-19. Such a difference has already been reported in the past with polyphenols, such as quercetin, especially in the context of oxidative stress caused by $\mathrm{H}_{2} \mathrm{O}_{2}$ [76]. The authors reported that many of the flavonoids were more effective at protecting primary RPE compared with ARPE-19. On this basis, it can be speculated that quercetin derivative $39 \mathrm{~b}$ can protect retinal cell types through different mechanisms, including direct scavenging of ROS, anti-apoptotic activity, and phase-2 induction [78]. It was also shown that the most effective compounds are more hydrophobic than quercetin, indicating that they should pass through cell membranes and accumulate intracellularly [79]. The DHA combined with quercetin should be consistent with an increase in hydrophobicity and cell bioavailability of the compound.

\section{Conclusions}

Among the research performed to develop pharmacological treatment for macular degeneration, most of the molecules have been discarded in the past because of toxicity issues. We propose here, a pharmacological approach based on a natural product ((poly)phenolic compound linked to omega-3 derivatives). Both parts of the molecule have already proven their beneficial effect in numerous studies as dietary complements: as (poly)phenols and omega-3 PUFAs are already present in our alimentation, the risk of toxicity compared to other synthetic drugs may be considerably reduced, as well as the unwanted side effects. Naturally, in vivo toxicity of lipophenols and their potential atRAL-adduct will have to be studied in future studies.

Starting from the phloroglucinol backbone, the present work evaluated the modification of the (poly)phenol core to achieve protection against both carbonyl and oxidative stresses in RPE cells. New synthetic routes were developed to access four original lipophenol series based on phloroglucinol, resveratrol, catechin and quercetin (poly)phenols. Despite cell-free assays proving aldehyde scavenging of natural (poly)
(A)

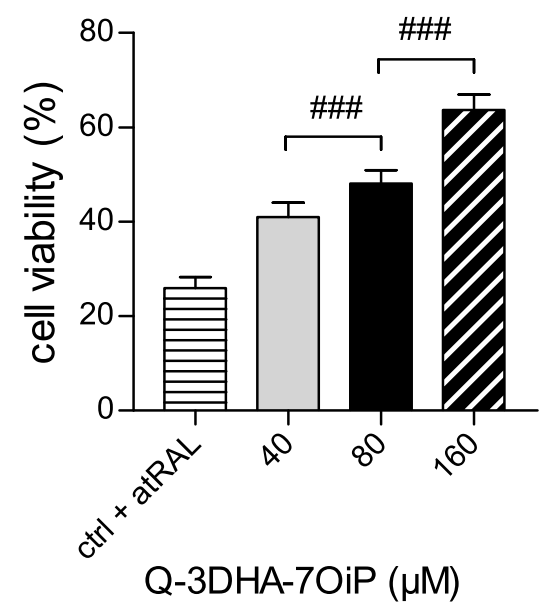

(B)

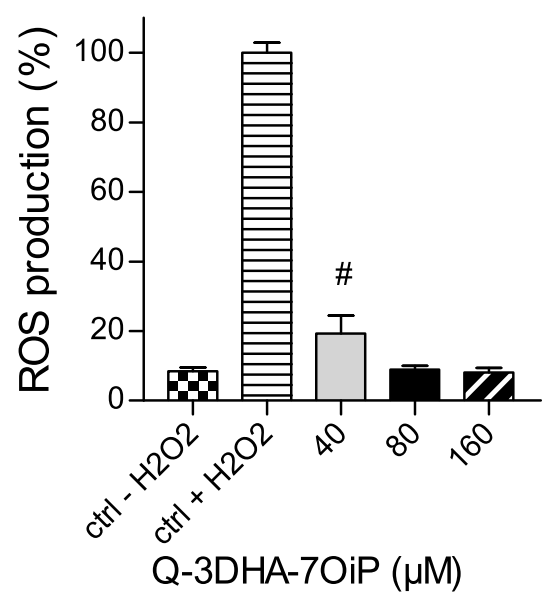

Fig. 14. Anti-COS activity of Q-3DHA7OiP (39b) on primary rat RPE cells. Results are expressed as mean \pm SEM and are from $n=3-5$ independent experiments. (A) Anti-carbonyl stress assay: cell viability (MTT) after incubation of Q-3DHA-7OiP (39b) $(0-160 \mu \mathrm{M})$ and atRAL $(25 \mu \mathrm{M})$. The data are expressed as the percentage of nontreated and non-exposed to atRAL control cells. All conditions have a p-value $<0.001$ versus non-treated and exposed to atRAL control cells. \#p $<0.05, \# \# \mathrm{p}$ $<0.01$, \#\#\#p $<0.001$, versus $80 \mu \mathrm{M}$ of lipophenol. (B) Antioxidant assay: representation of ROS production (DCFDA probe) after incubation of Q-3DHA7OiP (39b) $(0-160 \mu \mathrm{M})$ and $\mathrm{H}_{2} \mathrm{O}_{2}(600$ $\mu \mathrm{M})$ in primary cells. The data are expressed as the percentage of nontreated and exposed to $\mathrm{H}_{2} \mathrm{O}_{2}$ cells. All conditions have a p-value $<0.001$ versus non-treated and exposed to $\mathrm{H}_{2} \mathrm{O}_{2}$ control cells. \#p $<0.05, \# \# \mathrm{p}<0.01$, $\# \# \# \mathrm{p}<0.001$, versus non-treated and non-exposed to $\mathrm{H}_{2} \mathrm{O}_{2}$ cells. 
phenols, our work highlights that, in biological media, the protection against carbonyl stress (produced by atRAL toxicity) requires additional chemical modifications of natural (poly)phenol to promote cellular protection. Both isopropyl-resorcinol and PUFA moieties were essential for promising anti-carbonyl stress activity in RPE cells. Substitution of two phenolic functions lower antioxidant capacity, compared to native (poly)phenols, as reported in literature [22], however di-substituted alkyl-lipophenols still provide sufficient antioxidant activities to reduce intracellular ROS. Despite significant literature relating direct RCS trapping using (poly)phenols in cell-free assays, there is limited information on this direct interaction in complex biological systems. This work highlights the importance of cellular assays to validate anti-carbonyl stress potency of (poly)phenol conjugates: chemicals able to scavenge aldehyde in cell-free assays are not necessarily active in biological media, because of a lack of bioavailability or cell penetration, but also because of the importance of the $\mathrm{pH}$ in RCS scavenging (increased in basic conditions) [80,81]. It is therefore relevant to consider the $\mathrm{pH}$ dependency of the aldehyde trapping by (poly)phenol, as $\mathrm{pH}$ can vary from one cell compartment to another. The exact mechanism of alkyl-lipophenol protection against atRAL toxicity is currently under investigation and may be an association of 1) direct aldehyde scavenging, chemically or enzymatically catalyzed [82]; 2) activation of aldehyde detoxification enzymes, as the alkyl-phloroglucinol lipophenol LEAD B is able to activate Nrf2-Keap1 pathway [24] (also reported to activate aldehyde dehydrogenases and glutathione S-tranferases gene expression involved in aldehyde detoxification), and 3) reduction of ROS levels and oxidative stress induced by atRAL cell treatment [24,26,83].

Taken together, the different cellular assays performed on the ARPE19 cell line led to the discovery of an optimal DHA-quercetin anti-COS lipophenol (Q-3DHA-7OiP, 39b) showing optimized antioxidant properties compared to the previous phloroglucinol lead (LEAD B), and high protection against atRAL toxicity. Its anti-COS properties have also been validated in primary RPE cells. To conclude, Q-3DHA-7OiP was the most powerful lipophenol to suppress photo-oxidative toxicity initiated in RPE cells by A2E. Evaluation of photoreceptor protection against acute light-induced degeneration in the $\mathrm{Abca}^{-/-}$mouse model (involving both carbonyl and oxidative stresses), will be reported in due course using the best lipophenol candidate, Q-3DHA-7OiP.

\section{Experimental section}

\subsection{Chemical synthesis}

Experimental Procedures. The detailed discussion and the experimental procedures of chemical/enzymatic synthesis of all intermediates and final lipophenols are described in the supporting information (sections S1 to S7), as well as their full analytic characterization $\left({ }^{1} \mathrm{H}\right.$ and ${ }^{13} \mathrm{C}$ NMR, HRMS analysis, Rf, melting point). Known compounds were prepared according to previously described procedures: mono-isopropylphloroglucinol (1) and P-OiP-ODHA (LEAD B) [23], P-OiP-OLA (LEAD A) [24], R-4'LA and R-4'DHA [43], Q-3LA, Q-3DHA and C-3LA [42].

\subsection{Biological evaluations}

Chemicals. All lipophenols were dissolved in dimethylsulfoxide (DMSO) to prepare a stock solution at $80 \mathrm{mM}$. Hydrogen peroxide solution $\left(\mathrm{H}_{2} \mathrm{O}_{2}, 30\right.$ wt \% in $\left.\mathrm{H}_{2} \mathrm{O}\right)$, all-trans-retinal (atRAL) and 3-(4,5dimethylthiazol-2-yl)-2,5-diphenyl tetrazolium bromide (MTT) were purchased from Sigma-Aldrich. $\mathrm{N}$-retinylidene- $\mathrm{N}$-retinylethanolamine (A2E) was synthesized as previously described by Parish et al., in 1998 [84]. 2',7'-dichlorofluorescin diacetate (DCFDA) was purchased from Sigma-Aldrich (Saint-Quentin, France) and dissolved in DMSO to prepare stock solution at $20 \mathrm{mM}$. All stock solutions of lipophenols, atRAL, $\mathrm{A} 2 \mathrm{E}$ and probe were stored at $-20^{\circ} \mathrm{C}$ in the dark.

Cell Culture. ARPE-19 cells were obtained from ATCC (USA) and were grown in Dulbecco's Modified Eagle's Medium (DMEM)/Ham F12 (GIBCO) containing $10 \% \mathrm{v} / \mathrm{v}$ fetal bovine serum (FBS) and $1 \% \mathrm{v} / \mathrm{v}$ penicillin/streptomycin under atmospheric humidified air $(95 \%) / \mathrm{CO}_{2}$ (5\%) at $37{ }^{\circ} \mathrm{C}$. For experimental cell seeding and sub-culturing, the cells were dissociated with $0.25 \%$ trypsin-EDTA, resuspended in the culture medium and then plated at $1-3 \times 10^{5}$ cells $/ \mathrm{mL}$. ARPE- 19 cells were cultured and used up to 15 passages. Primary RPE cells were established from Long-Evans newborn rats according to the procedure described previously [26,77]. Briefly, after enucleation of the eyes, intact RPE sheets were separated from the choroid and dissociated in a trypsin-EDTA solution (GIBCO) to obtain a suspension of single cells. RPE cells, cultured in 96 -well plates, reached $80-85 \%$ of confluence after 3 days in atmospheric humidified air $(95 \%) / \mathrm{CO}_{2}(5 \%)$ at $37{ }^{\circ} \mathrm{C}$, and were used for cell assays without passaging.

Cell Viability. Cell viability was determined in ARPE-19 and primary rat RPE cells by MTT colorimetric assay. The cells were incubated for $2 \mathrm{~h}$ with MTT reagent $(0.5 \mathrm{mg} / \mathrm{mL})$. During this incubation time, mitochondrial dehydrogenases of the living cells reduce the MTT to insoluble purple formazan, which was then dissolved with DMSO to form a colored solution. The absorbance of supernatants, which was proportional to the number of living cells, was measured at $570 \mathrm{~nm}$ and $655 \mathrm{~nm}$ using a microplate reader (BioRad 550, USA or CLARIOstar Plus, BMG Labtech). The absorbance of the compounds tested does not interfere with the absorbance at 570 and $655 \mathrm{~nm}$. The percentage of the viable cells was calculated as [(OD570 sample-OD655 sample)/(OD570 control-OD655 control)] $\times 100 \%$.

Cytotoxicity of Lipophenols. ARPE-19 cells were plated into 96well plates $\left(4 \times 10^{4}\right.$ cells/well) and cultured for $24 \mathrm{~h}$ to reach confluence before lipophenol treatment. The cell cultures were treated with serum free medium containing the lipophenols at different concentrations $(0-160 \mu \mathrm{M})$ for $24 \mathrm{~h}$. Control cells were incubated with DMSO $(0.2 \%)$. The viability of the cells was determined using MTT colorimetric assay, as described above, and expressed as a percentage of viable cells normalized with control conditions in the absence of lipophenols.

Protection of Lipophenols against atRAL Toxicity. ARPE-19 cells were plated into 96 -well plates $\left(4 \times 10^{4}\right.$ cells/well) and cultured for $24 \mathrm{~h}$ to reach confluence before lipophenol treatment. The cell cultures were treated with serum free DMEM/F12 medium containing lipophenols at different concentrations $(0-80 \mu \mathrm{M})$ for $1 \mathrm{~h}$. Then atRAL was added to a final concentration of $15 \mu \mathrm{M}$ for $4 \mathrm{~h}$ before rinsing with medium. Control cells were incubated with DMSO $(0.2 \%) \pm$ atRAL. The cells incubated at $37{ }^{\circ} \mathrm{C}$ and viability was determined $16-20 \mathrm{~h}$ later using a MTT colorimetric assay. For primary rat RPE cultures, cells were treated with atRAL $(25 \mu \mathrm{M})$ in the presence of lipophenol $(40-160 \mu \mathrm{M})$ for $4 \mathrm{~h}$ before cell viability determination. Results are expressed in percentage of viable cells normalized with control conditions in the absence of lipophenol and stressor.

Impact of Lipophenols on ROS Level. ROS level was measured in $A R P E-19$ and primary rat RPE cells using dichlorofluorescein diacetate (DCFDA) reagent. The cell permeant reagent DCFDA is deacetylated by cellular esterases to dichlorofluorescein (DCFH2), which can be oxidized by several radical reactive species (peroxyl, alkoxyl, $\mathrm{NO}_{2}$, carbonate, $\mathrm{HO}, \ldots$ ) into the fluorophore $2^{\prime}, 7^{\prime}$-dichlorofluorescein (DCF) [85]. Intensity of fluorescence was measured during DCFDA oxidation by radical species to calculate level of radical reactive species. ARPE-19 cells were plated into black, optically clear flat bottom 96-well plates ( 4 $\times 10^{4}$ cells/well) and cultured for $24 \mathrm{~h}$ to reach confluence before the drug treatment. The cell cultures were incubated with $2 \mu \mathrm{M}$ of DCFDA for $45 \mathrm{~min}$ in DMEM/F12 medium without phenol red $+1 \%$ FBS. The cells were rinsed and incubated with the medium containing lipophenols at different concentrations $(0-80 \mu \mathrm{M})$ for $1 \mathrm{~h}$. Then, $\mathrm{H}_{2} \mathrm{O}_{2}$ was added to a final concentration of $600 \mu \mathrm{M}$ for $4 \mathrm{~h}$. Primary rat RPE cells were seeded on white, opaque-bottomed 96-well plates. On day 3, cells were incubated for $45 \mathrm{~min}$ at $37{ }^{\circ} \mathrm{C}$ in $1 \mathrm{X}$ Buffer containing $25 \mu \mathrm{M}$ of DCFDA. The cells were then treated with $600 \mu \mathrm{M}$ of $\mathrm{H}_{2} \mathrm{O}_{2}$ in the presence of lipophenol $(40-160 \mu \mathrm{M})$ for $4 \mathrm{~h}$ at $37{ }^{\circ} \mathrm{C}$. For both ARPE-19 and primary 
cells, DCF production was measured by fluorescence spectroscopy with excitation wavelength at $485 \mathrm{~nm}$ and emission wavelength at $535 \mathrm{~nm}$. The fluorescence of the compounds tested does not interfere with DCFDA signal. Control cells were incubated with DMSO $(0.2 \%) \pm$ DCFDA $\pm \mathrm{H}_{2} \mathrm{O}_{2}$. The percentage of ROS produced was calculated as [(fluorescence of sample)/(fluorescence of control)] $\times 100 \%$. The results are expressed in percentage of ROS produced normalized with control conditions in the absence of lipophenol and presence of $\mathrm{H}_{2} \mathrm{O}_{2}$.

Protection of Lipophenols against Photo-Oxidized A2E Toxicity. ARPE-19 cells were plated into 96-well plates $\left(4 \times 10^{4}\right.$ cells/well $)$ and cultured for $24 \mathrm{~h}$ to reach confluence before lipophenol treatment. The cell cultures were treated with serum free DMEM/F12 medium without phenol red containing lipophenols at different concentrations $(0-80 \mu \mathrm{M})$ for $1 \mathrm{~h}$. Then A2E was added to a final concentration of $20 \mu \mathrm{M}$ for $6 \mathrm{~h}$ before rinsing with medium. Control cells were incubated with DMSO $(0.2 \%) \pm$ A2E. The cells were exposed to intense blue light (4600 LUX) for $30 \mathrm{~min}$ to induce phototoxicity of A2E and incubated at $37^{\circ} \mathrm{C}$. The cell viability was determined $16-20 \mathrm{~h}$ later using a MTT colorimetric assay. Results are expressed as a percentage of viable cells normalized with control conditions in the absence of lipophenols and stressor. When a dose-dependent efficiency was observed, $\mathrm{EC}_{50}$ was calculated.

Statistical Analysis. The data are presented as means \pm SEM determined from at least three independent experiments. In each experiment, all conditions were done at least in quadruplicate. Statistical analyses were performed by Oneway ANOVA test using NewmanKeuls's post-hoc for Gaussian distributions (the normality of distributions was evaluated with a Shapiro-Wilk test) and differences with pvalues $<0.05$ were considered as statistically significant. $\mathrm{EC}_{50}$ were calculated using GraphPad Prism version 5.03 and non-linear regression.

\subsection{Associated content}

Full discussion on chemical synthesis of C-phloroglucinol derivatives (Section S1); Characterization of the silylated chromane orthoester byproduct of compound 5 (Section S2); Full discussion on chemical synthesis of resveratrol derivatives (Section S3); Full discussion on chemical synthesis of (+)-catechin derivatives (Section S4); Full discussion on chemical synthesis of quercetin-50iP derivatives (Section S5); Full discussion on chemical synthesis of quercetin-7OiP derivatives (Section S6); Experimental procedure and full analysis characterization of all intermediates and final compounds (2a to $39 \mathbf{b}$, Section S7); ${ }^{1} \mathrm{H}$ and ${ }^{13} \mathrm{C}$ NMR spectra for all intermediates and final compounds (2a to $39 \mathbf{b}$, Section S8).

\section{Funding sources}

This research was funded by University of Montpellier, SATT AxLR (181088BU) and ANR (LipoPheRet - ANR-18-CE18-0017). Inserm and CNRS are thanked for their support.

\section{CRediT authorship contribution statement}

Espérance Moine: Writing - original draft, Writing - review \& editing. Manel Boukhallat: Writing - original draft, Writing - review \& editing. David Cia: Writing - original draft, Writing - review \& editing. Nathalie Jacquemot: Writing - original draft, Writing - review \& editing. Laurent Guillou: Writing - original draft, Writing - review \& editing. Thierry Durand: Writing - original draft, Writing - review \& editing. Joseph Vercauteren: Writing - original draft, Writing - review \& editing. Philippe Brabet: Writing - original draft, Writing - review \& editing. Céline Crauste: Writing - original draft, Writing - review \& editing.

\section{Acknowledgments}

The authors would like to thank Dr. Gaetan Drouin and Pr. Vincent Rioux for providing DHA-ethyl ester necessary for lipophenol synthesis, and Dr. Vasiliki Kalatzis for English correction of the manuscript.

\section{Appendix A. Supplementary data}

Supplementary data to this article can be found online at https://doi. org/10.1016/j.freeradbiomed.2020.10.316.

\section{References}

[1] J.Z. Nowak, Oxidative stress, polyunsaturated fatty acids-derived oxidation products and bisretinoids as potential inducers of CNS diseases: focus on agerelated macular degeneration, Pharmacol. Rep. 65 (2013) 288-304.

[2] J.R. Sparrow, E. Gregory-Roberts, K. Yamamoto, A. Blonska, S.K. Ghosh, K. Ueda, J. Zhou, The bisretinoids of retinal pigment epithelium, Prog. Retin. Eye Res. 31 (2012) 121-135.

[3] J.T. Handa, M. Cano, L. Wang, S. Datta, T. Liu, Lipids, oxidized lipids, oxidationspecific epitopes, and Age-related Macular Degeneration, Biochim. Biophys. Acta 1862 (2017) 430-440.

[4] A.V. Cideciyan, T.S. Aleman, M. Swider, S.B. Schwartz, J.D. Steinberg, A. J. Brucker, A.M. Maguire, J. Bennett, E.M. Stone, S.G. Jacobson, Mutations in ABCA4 result in accumulation of lipofuscin before slowing of the retinoid cycle: a reappraisal of the human disease sequence, Hum. Mol. Genet. 13 (2004) 525-534.

[5] J.R. Sparrow, N. Fishkin, J. Zhou, B. Cai, Y.P. Jang, S. Krane, Y. Itagaki, K. Nakanishi, A2E, a byproduct of the visual cycle, Vis. Res. 43 (2003) 2983-2990.

[6] S.R. Kim, S. Jockusch, Y. Itagaki, N.J. Turro, J.R. Sparrow, Mechanisms involved in A2E oxidation, Exp. Eye Res. 86 (2008) 975-982.

[7] Y. Wu, E. Yanase, X. Feng, M.M. Siegel, J.R. Sparrow, Structural characterization of bisretinoid A2E photocleavage products and implications for age-related macular degeneration, Proc. Natl. Acad. Sci. U. S. A 107 (2010) 7275-7280.

[8] A.E. Sears, P.S. Bernstein, A.V. Cideciyan, C. Hoyng, P.C. Issa, K. Palczewski, P. J. Rosenfeld, S. Sadda, U. Schraermeyer, J.R. Sparrow, I. Washington, H.P. N. Scholl, Towards treatment of Stargardt disease: workshop organized and sponsored by the foundation fighting blindness, Transl, Vis. Sci. Technol. 6 (2017) $1-32$.

[9] D. Kook, A.H. Wolf, A.L. Yu, A.S. Neubauer, S.G. Priglinger, A. Kampik, U.C. WelgeLussen, The protective effect of quercetin against oxidative stress in the human RPE in vitro, Invest. Ophthalmol. Vis. Sci. 49 (2008) 1712-1720.

[10] R.E. King, K.D. Kent, J.A. Bomser, Resveratrol reduces oxidation and proliferation of human retinal pigment epithelial cells via extracellular signal-regulated kinase inhibition, Chem. Biol. Interact. 151 (2005) 143-149.

[11] W. Kalt, A. Hanneken, P. Milbury, F. Tremblay, Recent research on polyphenolics in vision and eye health, J. Agric. Food Chem. 58 (2010) 4001-4007.

[12] S. Quideau, D. Deffieux, C. Douat-Casassus, L. Pouységu, Plant polyphenols: chemical properties, biological activities, and synthesis, Angew. Chem. Int. Ed. 50 (2011) 586-621.

[13] H.J. Forman, K.J.A. Davies, F. Ursini, How do nutritional antioxidants really work: nucleophilic tone and para-hormesis versus free radical scavenging in vivo, Free Radic. Biol. Med. 66 (2014) 24-35.

[14] Y. Xie, X. Chen, Structures required of polyphenols for inhibiting advanced glycation end products formation, Curr. Drug Metabol. 14 (2013) 414-431.

[15] I. Sadowska-Bartosz, G. Bartosz, Prevention of protein glycation by natural compounds, Molecules 20 (2015) 3309-3334.

[16] C.Y. Lo, W.T. Hsiao, X.Y. Chen, Efficiency of trapping methylglyoxal by phenols and phenolic acids, J. Food Sci. 76 (2011) 90-96.

[17] X. Shao, H. Chen, Y. Zhu, R. Sedighi, C.-T. Ho, S. Sang, Essential structural requirements and additive effects for flavonoids to scavenge methylglyoxal, J. Agric. Food Chem. 62 (2014) 3202-3210.

[18] W. Wang, Y. Qi, J.R. Rocca, P.J. Sarnoski, A. Jia, L. Gu, Scavenging of toxic acrolein by resveratrol and hesperetin and identification of adducts, J. Agric. Food Chem. 63 (2015) 9488-9495.

[19] F. Yin, X. Wang, Y. Hu, H. Xie, X. Liu, L. Qin, J. Zhang, D. Zhou, F. Shahidi, Evaluation of absorption and plasma pharmacokinetics of tyrosol acyl esters in rats, J. Agric. Food Chem. 68 (2020) 1248-1256.

[20] O.M. Feeney, M.F. Crum, C.L. McEvoy, N.L. Trevaskis, H.D. Williams, C.W. Pouton, W.N. Charman, C.A.S. Bergström, C.J.H. Porter, 50 years of oral lipid-based formulations: provenance, progress and future perspectives, Adv. Drug Deliv. Rev. 101 (2016) 167-194.

[21] C.W. Pouton, Lipid formulations for oral administration of drugs: non-emulsifying, self-emulsifying and 'self-microemulsifying' drug delivery systems, Eur. J. Pharmaceut. Sci. 11 (2000) S93-S98.

[22] C. Crauste, M. Rosell, T. Durand, J. Vercauteren, Omega-3 polyunsaturated lipophenols, how and why? Biochimie 120 (2016) 62-74.

[23] C. Crauste, C. Vigor, P. Brabet, M. Picq, M. Lagarde, C. Hamel, T. Durand, J. Vercauteren, Synthesis and evaluation of polyunsaturated fatty acid-phenol conjugates as anti-carbonyl-stress lipophenols, Eur. J. Org Chem. (2014) 4548-4561.

[24] A. Cubizolle, D. Cia, E. Moine, N. Jacquemot, L. Guillou, M. Rosell, C. AngebaultProuteau, G. Lenaers, I. Meunier, J. Vercauteren, T. Durand, C. Crauste, P. Brabet, 
Isopropyl-phloroglucinol-DHA protects outer retinal cells against lethal dose of alltrans-retinal, J. Cell Mol. Med. 24 (2020) 5057-5069.

[25] T. Georgiou, A. Neokleous, D. Nicolaou, B. Sears, Pilot study for treating dry agerelated macular degeneration (AMD) with high-dose omega-3 fatty acids, PharmaNutrition 2 (2014) 8-11.

[26] D. Cia, A. Cubizolle, C. Crauste, N. Jacquemot, L. Guillou, C. Vigor, C. Angebault, C.P. Hamel, J. Vercauteren, P. Brabet, Phloroglucinol protects retinal pigment epithelium and photoreceptor against all-trans-retinal-induced toxicity and inhibits A2E formation, J. Cell Mol. Med. 20 (2016) 1651-1663.

[27] M. Suh, A.A. Wierzbicki, E. Lien, M.T. Clandinin, Relationship between dietary supply of long-chain fatty acids and membrane composition of long- and very long chain essential fatty acids in developing rat photoreceptors, Lipids 31 (1996) 61-64.

[28] P.K. Mukherjee, V.L. Marcheselli, C.N. Serhan, N.G. Bazan, Neuroprotectin D1: a docosahexaenoic acid-derived docosatriene protects human retinal pigment epithelial cells from oxidative stress, Proc. Natl. Acad. Sci. U. S. A 101 (2004) 8491-8496.

[29] P. Brabet, A. Cubizolle, C. Crauste, L. Guillou, A.L. Bonnefont, J. Vercauteren, T. Durand, C.P. Hamel, A phloroglucinol-DHA derivative protects against lightinduced retinal degeneration in an Abca4-deficient mouse model, Invest. Ophthalmol. Vis. Sci. 58 (2017) 2031.

[30] N. Taveau, A. Cubizolle, L. Guillou, N. Pinquier, E. Moine, D. Cia, J. Vercauteren, T. Durand, C. Crauste, P. Brabet, Preclinical pharmacology of a lipophenol in a mouse model of light-induced retinopathies, Exp. Mol. Med. 52 (2020) 1090-1101.

[31] Q. Zhu, Z.-P. Zheng, K.-W. Cheng, J.-J. Wu, S. Zhang, Y.S. Tang, K.-H. Sze, J. Chen, F. Chen, M. Wang, Natural polyphenols as direct trapping agents of lipid peroxidation-derived acrolein and 4-hydroxy-trans-2-nonenal, Chem. Res. Toxicol. 22 (2009) 1721-1727.

[32] W. Wang, Antiglycation Effects and Reactive Carbonyl Trapping Capacity of Berry and Grape Phytochemicals, Thesis of master of sciences of University of Florida, 2010, pp. 1-129.

[33] A. Alaimo, M.C. Di Santo, A.P. Domínguez Rubio, G. Chaufan, G. García Liñares, O. E. Pérez, Toxic effects of A2E in human ARPE-19 cells were prevented by resveratrol: a potential nutritional bioactive for age-related macular degeneration treatment, Arch. Toxicol. 94 (2020) 553-572.

[34] Y. Wang, H.J. Kim, J.R. Sparrow, Quercetin and cyanidin-3-glucoside protect against photooxidation and photodegradation of A2E in retinal pigment epithelia cells, Exp. Eye Res. 160 (2017) 45-55.

[35] W. Li, Y. Jiang, T. Sun, X. Yao, X. Sun, Supplementation of procyanidins B2 attenuates photooxidation-induced apoptosis in ARPE-19 cells, Int. J. Food Sci. Nutr. 67 (2016) 650-659.

[36] O. Gia, A. Anselmo, M.T. Conconi, C. Antonello, E. Uriarte, S. Caffieri, 4'-Methyl derivatives of 5-MOP and 5-MOA: synthesis, photoreactivity, and photobiologica activity, J. Med. Chem. 39 (1996) 4489-4496.

[37] M.J. So, E.J. Cho, Phloroglucinol attenuates free radical-induced oxidative stress, Prev. Nutr. Food Sci. 19 (2014) 129-135.

[38] M. Vestergaard, H. Ingmer, Antibacterial and antifungal properties of resveratrol, Int. J. Antimicrob. Agents 53 (2019) 716-723.

[39] J.-H. Kang, S.-Y. Choung, Protective effects of resveratrol and its analogs on agerelated macular degeneration in vitro, Arch Pharm. Res. (Seoul) 39 (2016) 1703-1715.

[40] A. Lançon, R. Frazzi, N. Latruffe, Anti-oxidant, anti-inflammatory and antiangiogenic properties of resveratrol in ocular diseases, Molecules 21 (2016) 1-8.

[41] I.C. Vlachogianni, E. Fragopoulou, I.K. Kostakis, S. Antonopoulou, In vitro assessment of antioxidant activity of tyrosol, resveratrol and their acetylated derivatives, Food Chem. 177 (2015) 165-173.

[42] E. Moine, P. Brabet, L. Guillou, T. Durand, J. Vercauteren, C. Crauste, New lipophenol antioxidants reduce oxidative damage in retina pigment epithelial cells, Antioxidants 7 (2018) 1-18.

[43] A. Shamseddin, C. Crauste, E. Durand, P. Villeneuve, G. Dubois, T. Pavlickova, T. Durand, J. Vercauteren, F. Veas, Resveratrol-Linoleate protects from exacerbated endothelial permeability via a drastic inhibition of the MMP-9 activity, Biosci. Rep. (2018) 1-13.

[44] C. Cren-Olivé, S. Lebrun, C. Rolando, An efficient synthesis of the four mono methylated isomers of $(+)$-catechin including the major metabolites and of some dimethylated and trimethylated analogues through selective protection of the catechol ring, J. Chem. Soc. Perkin 1 (2002) 821-830.

[45] Y. Shen, Z. Xu, Z. Sheng, Ability of resveratrol to inhibit advanced glycation end product formation and carbohydrate-hydrolyzing enzyme activity, and to conjugate methylglyoxal, Food Chem. 216 (2017) 153-160.

[46] S.-J. Sheu, N.-C. Liu, C.-C. Ou, Y.-S. Bee, S.-C. Chen, H.-C. Lin, J.Y.H. Chan, Resveratrol stimulates mitochondrial bioenergetics to protect retinal pigment epithelial cells from oxidative damage, Invest. Ophthalmol. Vis. Sci. 54 (2013) 6426-6438

[47] K.E. Heim, A.R. Tagliaferro, D.J. Bobilya, Flavonoid antioxidants: chemistry, metabolism and structure-activity relationships, J. Nutr. Biochem. 13 (2002) 572-584.

[48] S.D.S. Banjarnahor, N. Artanti, Antioxidant properties of flavonoids, Med. J. Indones. 23 (2015) 239-244.

[49] R. Khursheed, S.K. Singh, S. Wadhwa, M. Gulati, A. Awasthi, Enhancing the potential preclinical and clinical benefits of quercetin through novel drug delivery systems, Drug Discov. Today 25 (2020) 209-222.

[50] H. Kalantari, H. Foruozandeh, M.J. Khodayar, A. Siahpoosh, N. Saki, P. Kheradmand, Antioxidant and hepatoprotective effects of Capparis spinosa L. fractions and Quercetin on tert-butyl hydroperoxide- induced acute liver damage in mice, J. Tradit. Complement. Med. 8 (2018) 120-127.
[51] A.J. Dugas, J. Castañeda-Acosta, G.C. Bonin, K.L. Price, N.H. Fischer, G. W. Winston, Evaluation of the total peroxyl radical-scavenging capacity of Flavonoids: Structure-Activity relationships, J. Nat. Prod. 63 (2000) 327-331.

[52] K.L. Wolfe, R.H. Liu, Structure-Activity relationships of flavonoids in the cellular antioxidant activity assay, J. Agric. Food Chem. 56 (2008) 8404-8411.

[53] S. Hong, S. Liu, Targeted acylation for all the hydroxyls of $(+)$-catechin and evaluation of their individual contribution to radical scavenging activity, Food Chem. 197 (2016) 415-421.

[54] N.I. Howard, M.V.B. Dias, F. Peyrot, L. Chen, M.F. Schmidt, T.L. Blundell, C. Abell, Design and structural analysis of aromatic inhibitors of type II dehydroquinase from Mycobacterium tuberculosis, ChemMedChem 10 (2015) 116-133.

[55] S. Uesato, K. Taniuchi, A. Kumagai, Y. Nagaoka, R. Seto, Y. Hara, H. Tokuda, H. Nishino, Inhibitory effects of 3-O-acyl-(+)-catechins on Epstein-Barr virus activation, Chem. Pharm. Bull. 51 (2003) 1448-1450.

[56] X. Peng, K.-W. Cheng, J. Ma, B. Chen, C.-T. Ho, C. Lo, F. Chen, M. Wang, Cinnamon bark proanthocyanidins as reactive carbonyl scavengers to prevent the formation of advanced glycation endproducts, J. Agric. Food Chem. 56 (2008) 1907-1911.

[57] C.-Y. Lo, S. Li, D. Tan, M.-H. Pan, S. Sang, C.-T. Ho, Trapping reactions of reactive carbonyl species with tea polyphenols in simulated physiological conditions, Mol. Nutr. Food Res. 50 (2006) 1118-1128.

[58] M. Li, X. Han, B. Yu, Facile synthesis of flavonoid 7- O -glycosides, J. Org. Chem. 68 (2003) 6842-6845.

[59] B. Yang, R. Li, T. Woo, J.D. Browning, H. Song, Z. Gu, J. Cui, J.C. Lee, K.L. Fritsche, D.Q. Beversdorf, G.Y. Sun, C.M. Greenlief, Maternal dietary docosahexaenoic acid alters lipid peroxidation products and (n-3)/(n-6) fatty acid balance in offspring mice, Metabolites 9 (2019) 1-14.

[60] Y. Huang, W. Li, A.-N.T. Kong, Anti-oxidative stress regulator NF-E2-related factor 2 mediates the adaptive induction of antioxidant and detoxifying enzymes by lipid peroxidation metabolite 4-hydroxynonenal, Cell Biosci. 2 (2012) 1-7.

[61] A. Ishikado, K. Morino, Y. Nishio, F. Nakagawa, A. Mukose, Y. Sono, N. Yoshioka, K. Kondo, O. Sekine, T. Yoshizaki, S. Ugi, T. Uzu, H. Kawai, T. Makino, T. Okamura, M. Yamamoto, A. Kashiwagi, H. Maegawa, 4-Hydroxy hexenal derived from docosahexaenoic acid protects endothelial cells via Nrf2 activation, PLoS One 8 (2013) 1-13.

[62] I. Johansson, V.T. Monsen, K. Pettersen, J. Mildenberger, K. Misund, K. Kaarniranta, S. Schønberg, G. Bjørkøy, The marine n-3 PUFA DHA evokes cytoprotection against oxidative stress and protein misfolding by inducing autophagy and NFE2L2 in human retinal pigment epithelial cells, Autophagy 11 (2015) 1636-1651.

[63] Z. Sun, X. Peng, J. Liu, K.-W. Fan, M. Wang, F. Chen, Inhibitory effects of microalgal extracts on the formation of advanced glycation endproducts (AGEs), Food Chem. 120 (2010) 261-267.

[64] M. Odjakova, E. Popova, M.A. Sharif, R. Mironova, Plant-derived agents with antiglycation activity, in: S. Petrescu (Ed.), Glycosylation, chapter 10, IntechOpen Publishing, 2012, pp. 223-256.

[65] S.A. Belal, A.S. Sivakumar, D.R. Kang, S. Cho, H.S. Choe, K.S. Shim, Modulatory effect of linoleic and oleic acid on cell proliferation and lipid metabolism gene expressions in primary bovine satellite cells, Anim. Cell Syst. 22 (2018) 324-333.

[66] N.P. Rotstein, L.E. Politi, O.L. German, R. Girotti, Protective effect of docosahexaenoic acid on oxidative stress-induced apoptosis of retina photoreceptors, Invest. Ophthalmol. Vis. Sci. 44 (2003) 2252-2259.

[67] J.P. SanGiovanni, E.Y. Chew, The role of omega-3 long-chain polyunsaturated fatty acids in health and disease of the retina, Prog. Retin. Eye Res. 24 (2005) 87-138.

[68] E.E. Martínez Leo, R.A. Rojas Herrera, M.R. Segura Campos, Protective effect of omega 3 fatty acids EPA and DHA in the neurodegenerative disease, in: J.M. Mérillon, K.G. Ramawat (Eds.), Bioact. Mol. Food, Springer International Publishing, Cham, 2018, pp. 1-17.

[69] M. Tachikawa, S. Akanuma, T. Imai, S. Okayasu, T. Tomohiro, Y. Hatanaka, K. Hosoya, Multiple cellular transport and binding processes of unesterified docosahexaenoic acid in outer blood-retinal barrier retinal pigment epithelial cells, Biol. Pharm. Bull. 41 (2018) 1384-1392.

[70] C. Papuc, G.V. Goran, C.N. Predescu, V. Nicorescu, G. Stefan, Plant polyphenols as antioxidant and antibacterial agents for shelf-life extension of meat and meat products: classification, structures, sources, and action mechanisms, Compr. Rev. Food Sci. Food Saf. 16 (2017) 1243-1268.

[71] J.R. Sparrow, H.R. Vollmer-Snarr, J. Zhou, Y.P. Jang, S. Jockusch, Y. Itagaki, K. Nakanishi, A2E-epoxides damage DNA in retinal pigment epithelial cells, J. Biol. Chem. 278 (2003) 18207-18213.

[72] M. Wrona, M. Różanowska, T. Sarna, Zeaxanthin in combination with ascorbic acid or $\alpha$-tocopherol protects ARPE-19 cells against photosensitized peroxidation of lipids, Free Radic. Biol. Med. 36 (2004) 1094-1101.

[73] V.M. Parmar, T. Parmar, E. Arai, L. Perusek, A. Maeda, A2E-associated cell death and inflammation in retinal pigmented epithelial cells from human induced pluripotent stem cells, Stem Cell Res. 27 (2018) 95-104.

[74] S. Ben-Shabat, Y. Itagaki, S. Jockusch, J.R. Sparrow, N.J. Turro, K. Nakanishi, formation of a nonaoxirane from $\mathrm{A} 2 \mathrm{E}$, a lipofuscin fluorophore related to macular degeneration, and evidence of singlet oxygen involvement, Angew. Chem. Int. Ed. 41 (2002) 814-817.

[75] A.V. Kuznetsova, A.M. Kurinov, M.A. Aleksandrova, Cell models to study regulation of cell transformation in pathologies of retinal pigment epithelium, J. Ophthalmol. (2014) 1-18.

[76] A. Hanneken, F.-F. Lin, J. Johnson, P. Maher, Flavonoids protect human retinal pigment epithelial cells from oxidative-stress-induced death, Invest. Ophthalmol. Vis. Sci. 47 (2006) 3164-3177. 
[77] D. Cia, J. Vergnaud-Gauduchon, N. Jacquemot, M. Doly, Epigallocatechin gallate (EGCG) prevents $\mathrm{H}_{2} \mathrm{O}_{2}$-induced oxidative stress in primary rat retinal pigment epithelial cells, Curr. Eye Res. 39 (2014) 944-952.

[78] E. Pawlowska, J. Szczepanska, A. Koskela, K. Kaarniranta, J. Blasiak, Dietary polyphenols in age-related macular degeneration: protection against oxidative stress and beyond, Oxid. Med. Cell. Longev. 2019 (2019) 1-13.

[79] K. Ishige, D. Schubert, Y. Sagara, Flavonoids protect neuronal cells from oxidative stress by three distinct mechanisms, Free Radic. Biol. Med. 30 (2001) 433-446.

[80] H. Zhu, M.M. Poojary, M.L. Andersen, M.N. Lund, Effect of pH on the reaction between naringenin and methylglyoxal: a kinetic study, Food Chem. 298 (2019) $1-9$.

[81] S. Sang, X. Shao, N. Bai, C.-Y. Lo, C.S. Yang, C.-T. Ho, Tea polyphenol (-)-Epigallocatechin-3-Gallate: a new trapping agent of reactive dicarbonyl species, Chem. Res. Toxicol. 20 (2007) 1862-1870.
[82] B. Szwergold, Reactions between methylglyoxal and its scavengers in-vivo appear to be catalyzed enzymatically, Med, Hypotheses 109 (2017) 153-155.

[83] Y. Chen, K. Okano, T. Maeda, V. Chauhan, M. Golczak, A. Maeda, K. Palczewski, Mechanism of all- trans -retinal toxicity with implications for Stargardt disease and age-related macular degeneration, J. Biol. Chem. 287 (2012) 5059-5069.

[84] C.A. Parish, M. Hashimoto, K. Nakanishi, J. Dillon, J. Sparrow, Isolation and onestep preparation of A2E and iso-A2E, fluorophores from human retinal pigment epithelium, Proc. Natl. Acad. Sci. U. S. A 95 (1998) 14609-14613.

[85] B. Halliwell, M. Whiteman, Measuring reactive species and oxidative damage in vivo and in cell culture: how should you do it and what do the results mean? Br. J. Pharmacol. 142 (2004) 231-255. 